1

2

3

4

5

6

7

8

9

$10{ }^{1}$ Department of Otorhinolaryngology, Perelman School of Medicine, University of

11 Pennsylvania, United States

$12{ }^{2}$ Department of Neuroscience, Perelman School of Medicine, University of Pennsylvania,

13 United States

14 * For correspondence: ntardiff@sas.upenn.edu

$15 * *$ Contributed equally

\section{and physiological mechanisms}

\author{
Nathan Tardiff ${ }^{*}$ \\ Lalitta Suriya-Arunroj $^{1}$ \\ Yale E. Cohen ${ }^{1 * *}$ \\ Joshua I. Gold ${ }^{\text {*** }}$
}

16 


\section{Abstract}

18 The varied effects of expectations on auditory perception are not well understood. For example,

19 both top-down rules and bottom-up stimulus regularities generate expectations that can bias

20 subsequent perceptual judgments. However, it is unknown whether these different sources of

21 bias use the same or different computational and physiological mechanisms. We examined how

22 rule-based and stimulus-based expectations influenced human subjects' behavior and pupil-

23 linked arousal, a marker of certain forms of expectation-based processing, during an auditory

24 frequency-discrimination task. Rule-based cues biased choice and response times (RTs) toward

25 the more-probable stimulus. In contrast, stimulus-based cues had a complex combination of

26 effects, including choice and RT biases toward and away from the frequency of recently heard

27 stimuli. These different behavioral patterns also had distinct computational signatures, including

28 different modulations of key components of a novel form of a drift-diffusion model, and distinct

29 physiological signatures, including substantial bias-dependent modulations of pupil size in

30 response to rule-based but not stimulus-based cues. These results imply that different sources of

31 expectations can modulate auditory perception via distinct mechanisms: one that uses arousal-

32 linked, rule-based information and another that uses arousal-independent, stimulus-based

33 information to bias the speed and accuracy of auditory perceptual decisions. 


\section{Introduction}

The auditory system is sensitive to expectations (Chambers et al., 2017; Gifford, Cohen, \&

Stocker, 2014; Heilbron \& Chait, 2018). Some expectations are instructed explicitly, such as via cues that carry information about the probability of occurrence of subsequent auditory stimuli.

Other expectations are inferred explicitly or implicitly from the features of an auditory stimulus, including temporal or structural regularities. Despite the importance of both rule-based and stimulus-based expectations, we lack basic knowledge about the effects of both forms of expectations on auditory perception, including whether they use distinct or shared computational and physiological mechanisms.

One set of open questions relates to our understanding of how rule-based and stimulus-based expectations affect categorical auditory decisions. In general, for non-auditory decision tasks, rule-based expectations can lead to biases in behavioral choices and response times (RTs) that are consistent with normative principles, including a tendency to make faster and more-prevalent choices to more-probable alternatives (Dunovan, Tremel, \& Wheeler, 2014; Hanks, Mazurek, Kiani, Hopp, \& Shadlen, 2011; Kelly, Corbett, \& O’Connell, 2020; Mulder, Wagenmakers, Ratcliff, Boekel, \& Forstmann, 2012). These effects are thought to be mediated via top-down input from higher-order brain regions to earlier sensory regions (Summerfield \& de Lange, 2014). However, the role of such rule-based expectations in auditory decision-making is relatively unexplored (except see de Gee et al., 2020; Gifford et al., 2014).

In contrast, stimulus-based expectations have been studied extensively and are thought to reflect, in part, bottom-up mechanisms in the auditory system, such as neural adaptation to stimulus regularities (Chambers et al., 2017; Lesicko, Angeloni, Blackwell, De Biasi, \& Geffen, 2021; Nelken, 2014; Parras et al., 2017; Todorovic \& de Lange, 2012; Todorovic, van Ede, Maris, \& 
57 de Lange, 2011). These adaptation-like mechanisms can have the opposite effect as rule-based cues, potentiating responses to violations of stimulus regularities and biasing perception away from recent stimuli (Alais, Orchard-Mills, \& Van der Burg, 2015; Holt \& Lotto, 2008; Shu,

60 Swindale, \& Cynader, 1993; Stocker \& Simoncelli, 2005). However, it is also feasible that

61 stimulus-based cues could be used to rapidly update prior beliefs, which could then bias

62 subsequent decisions via the same top-down mechanisms used for rule-based effects (Bastos,

63 Lundqvist, Waite, Kopell, \& Miller, 2020; Bastos et al., 2012; de Lange, Heilbron, \& Kok, 2018;

64 Fischer \& Whitney, 2014; Heilbron \& Chait, 2018; Krishnamurthy, Nassar, Sarode, \& Gold,

65 2017; Sotiropoulos, Seitz, \& Seris, 2011; Todorovic et al., 2011; Tsunada, Cohen, \& Gold, 66 2019).

67 We thus sought to answer a basic question that is fundamental to our understanding of how the

68 brain uses rule-based and stimulus-based information to form auditory decisions: do decision biases elicited by explicit top-down rules versus those elicited by stimulus regularities use the same or different computational and physiological mechanisms? To answer this question, we recruited human subjects to perform a two-alternative, forced-choice frequency-discrimination task. These subjects reported whether a test stimulus (a tone burst that was embedded in a noisy background) was "low frequency" or "high frequency." We manipulated both the signal-to-noise ratio (SNR) of the test stimulus (relative to the background) and two types of expectationgenerating cues: 1) rule-based cues, in the form of visual stimuli indicating the probability of each test stimulus; and 2) stimulus-based cues, in the form of temporal sequences of tone bursts, akin to those used to study stimulus-specific adaptation and mismatch negativity (Näätänen, Paavilainen, Rinne, \& Alho, 2007; Nelken, 2014; Sussman, 2007), that immediately preceded presentation of the test stimulus. We also measured the subjects' pupil diameter, which is a 
80 physiological marker of arousal that is sensitive to certain cognitive processes related to

81 decision-making (Joshi \& Gold, 2020), including decision biases (de Gee et al., 2017; de Gee,

82 Knapen, \& Donner, 2014; de Gee et al., 2020; Krishnamurthy et al., 2017; Urai, Braun, \&

83 Donner, 2017) and violations of top-down, but not bottom-up, expectations (Filipowicz, Glaze,

84 Kable, \& Gold, 2020). We found that rule-based and stimulus-based behavioral biases exhibited

85 distinct computational and physiological (i.e., pupillometric) signatures that may reflect

86 differential contributions of top-down and bottom-up forms of expectation-dependent

87 information processing in auditory perceptual decision-making. 
a

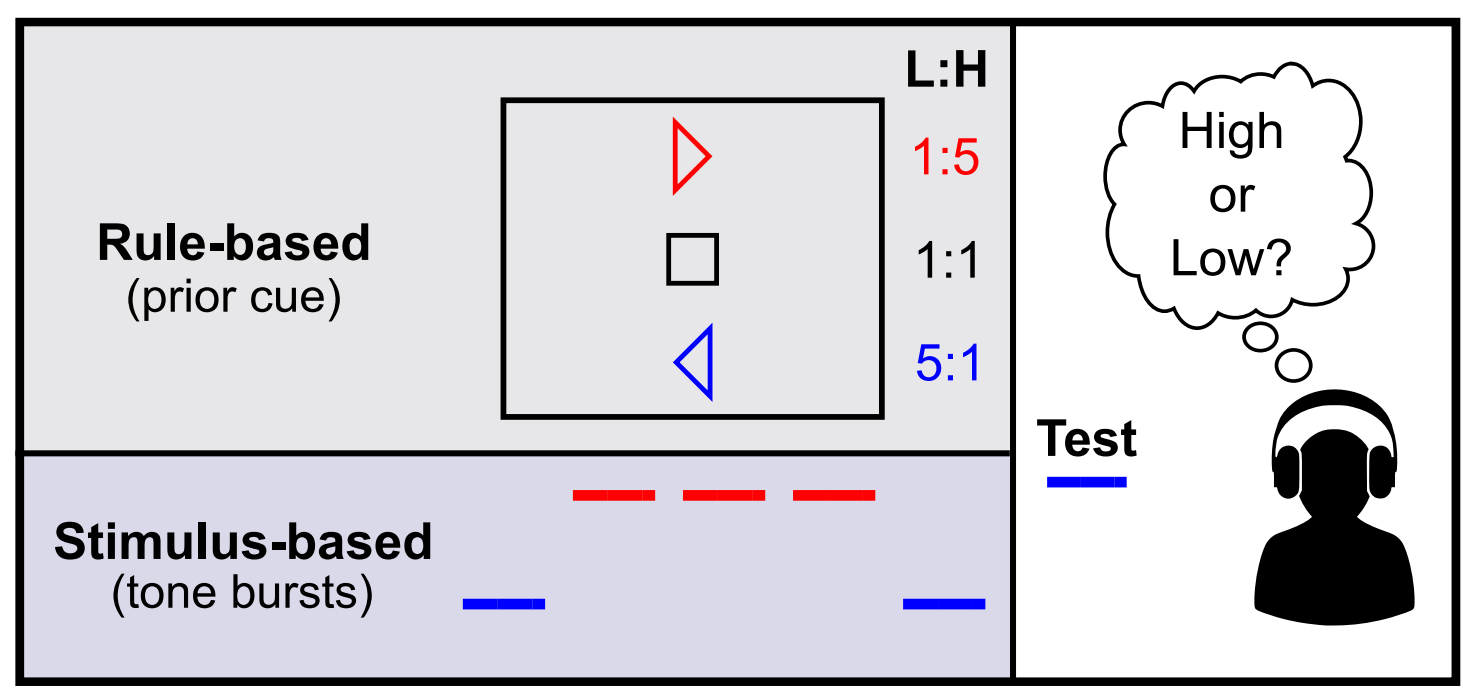

b

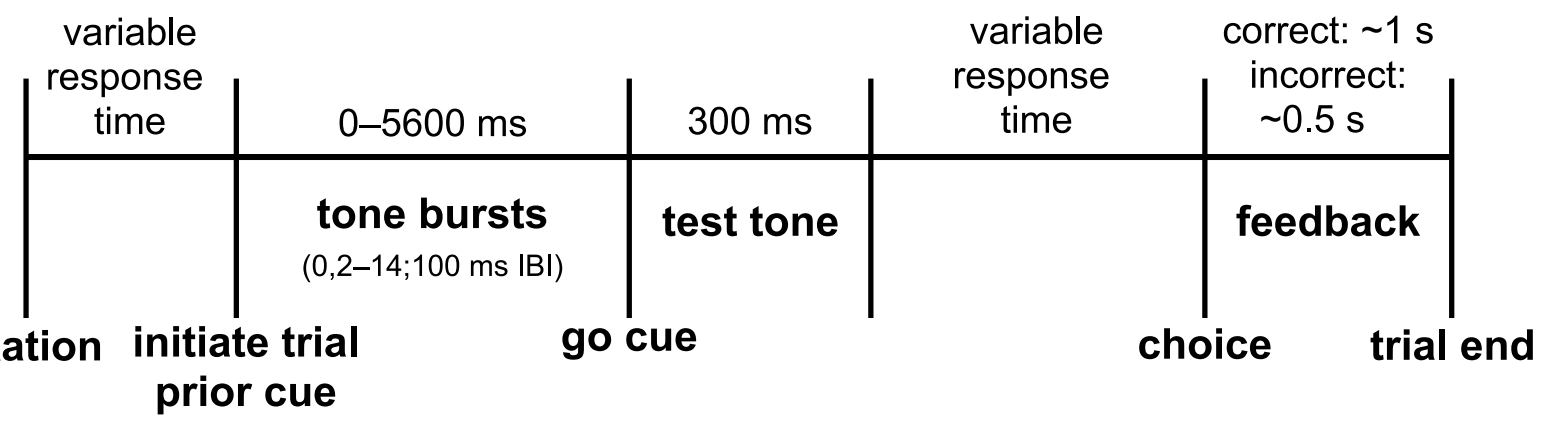

Figure 1. Task structure. a Subjects indicated with a button press whether a test tone was "high frequency" or "low frequency." In the rule-based condition, the test tone was preceded by a visual stimulus (prior cue) that indicated the probability that the upcoming tone would be low (L) or high $(\mathrm{H})$ frequency, with the probability determined by the L:H ratio. In the stimulus-based condition, the test tone was preceded by a variable-length, temporal "pre-test" sequence of lowand high-frequency tone bursts. On average, this sequence was not predictive of the test tone. In the mixed conditions, subjects received both rule-based and stimulus-based cues on each trial. b

96 Detailed trial timing. After attaining fixation, the subject initiated the trial with a button press.

97 The subject was then able to report their decision with a second button press at any time

98 following the "go cue" (up to 2000-3000 ms). 


\section{Results}

101 Fifty human subjects performed a frequency-discrimination task in which they indicated with a

102 button press whether a test stimulus was high frequency or low frequency (Figure 1). Task

103 difficulty was titrated by embedding the test stimulus in a background of white noise of various

104 signal-to-noise ratios (SNRs). We manipulated prior information in a blockwise fashion for each

105 subject. In the rule-based condition, prior to stimulus onset (Figure 1), one of three visual cues

106 indicated the probability that the upcoming test stimulus would be low frequency or high

107 frequency (high:low ratio = 1:5 (low), 1:1 (neutral), or 5:1 (high)). In the stimulus-based

108 condition, prior information was manipulated by presenting a temporal sequence of tone bursts

109 (using the maximum SNR; 2-14 bursts per trial) prior to the test stimulus. This "pre-test"

110 sequence varied in terms of its pattern of low and high frequencies but, on average, was not

111 predictive of the frequency of the test tone. We also presented mixed conditions in which

112 subjects encountered both rule-based and stimulus-based cues on each trial. 

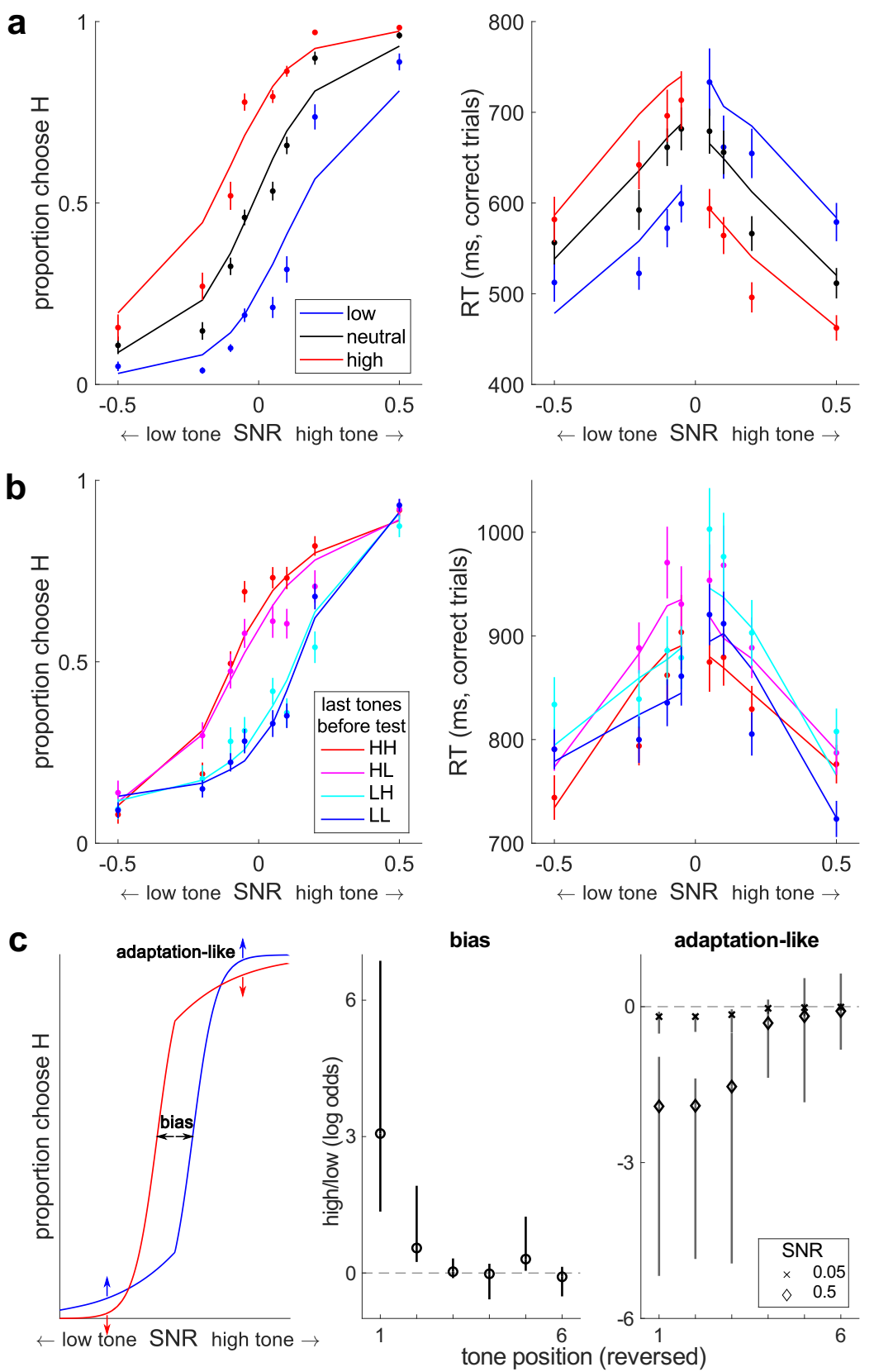

115 Figure 2. Behavioral summary. a Psychometric (left) and chronometric (right) functions from the rule-based condition for each prior cue (see legend). b Psychometric (left) and chronometric

117 (right) functions from the stimulus-based condition for different patterns of the last two pre-test 118 tone bursts (see legend; $\mathrm{HH}=$ the last two tones were high, $\mathrm{HL}=$ the last tone was high and the 119 second-to-last tone was low, etc.). In $\mathbf{a}$ and $\mathbf{b}$, data points are averages over all subjects, and error bars are standard errors of the mean (SEM). Solid lines are DDM fits (see text). By convention,

121 positive and negative SNRs are low-frequency and high-frequency test tones, respectively. c 
122 Left: Schematic illustrating how choice effects in the stimulus-based condition can be

123 decomposed into: 1) a bias that shifts the psychometric function in favor of the most-recently

124 heard tones, which dominates at low absolute SNRs; and 2) an adaptation-like effect that

125 decreases sensitivity to the most-recently heard tones, which becomes prominent at high absolute

126 SNRs. Blue and red curves illustrate simulated psychometric functions corresponding to trials in

127 which the last pre-test tone burst was low or high frequency, respectively. Right: Logistic

128 analysis of the bias and adaptation-like effects in the stimulus-based condition, as a function of

129 pre-test tone-burst position ( $1=$ the tone just prior to the test tone, etc.). Values along the

130 ordinate are the median effect of a pre-test tone at that position on choice, measured as log-odds

131 ratios for high/low frequency (positive/negative values imply that a high-frequency tone at the

132 given position increases/decreases the odds of choosing high-frequency relative to low-frequency

133 tones). Error bars are bootstrapped 95\% confidence intervals (10,000 samples). 


\section{Rule- and stimulus-based biases had different effects on choices and RTs}

136

137

138

139

140

141

142

143

144

145

146

147

148

149

150

151

152

153

154

155

156

157

In the rule-based condition, the subjects' choices and RTs showed consistent biases in accordance with the prior cues (Figure 2a). In particular, the subjects tended to choose the moreprobable option more often and more quickly than a neutral or less-probable option. For example, when the prior cue indicated that the test tone was more likely to be high frequency, subjects chose high frequency more often and responded faster. To quantify these effects, we fit logistic and linear models to the subjects' choices and mean RTs from correct trials, respectively. The choice bias was well-characterized as an additive shift (bias) of the psychometric function in favor of the prior (mean $\triangle \mathrm{AIC}$ no bias versus bias $=54.60$ ). These fits were only marginally improved by allowing the slope (sensitivity) of the psychometric function to vary with the prior (mean $\triangle \mathrm{AIC}$ bias vs. bias + sensitivity $=0.29)$. A Bayesian random-effects analysis confirmed the bias-only model as the most likely model (protected exceedance probability $=0.82$ ).

The RTs included a sharp discontinuity within the low- and high-prior conditions depending on whether subjects chose with ("congruent"; i.e., the prior and choice were toward the same frequency) or against ("incongruent"; i.e., the prior and choice were toward opposite frequencies) the cued prior (modulation by congruence, $F(2,65.08)=160.43, p<0.0001$ ). For example, on average, RTs at the lowest SNR were $>100-\mathrm{ms}$ faster when subjects chose high frequency with a high-prior cue or low frequency with a low-prior cue than when they made a choice incongruent with the prior (Figure 2a, right). Congruent RTs were faster than both incongruent RTs $\left(B=128.17, t(226.27)=17.83 p_{\text {corrected }}<0.0001\right)$ and RTs on neutral prior trials $\left(B=75.48, t(51.03)=9.19, p_{\text {corrected }}<0.0001\right)$, whereas incongruent RTs were slower than neutral-prior RTs $\left(B=-52.69, t(49.30)=-6.04, p_{\text {corrected }}<0.0001\right)$. Thus, the prior cues resulted in choices that, on average, were more common and faster to the more probable alternative. 
In the stimulus-based condition, the subjects' choices and RTs showed a more complex pattern. Figure $2 \mathrm{~b}$ shows choices and RTs plotted as a function of the last two pre-test tone bursts for a given trial. At low SNRs of the test stimulus, choices were biased toward the frequency of the most-recently heard tones, and RTs were faster when the choice was congruent with the mostrecently heard tone. These effects are evident in the lateral shifts in the psychometric function and discontinuities (vertical shifts) in the RTs at low SNRs and are consistent with the rule-based effects (see Figure 2a). In contrast, at higher test SNRs, an adaptation-like effect was more prominent, such that subjects were, on average, more likely and faster to respond with the alternative that was incongruent with the most-recently heard tones. For example, subjects were more likely and faster to respond "high frequency" after having heard two low-frequency tones than after having heard two high-frequency tones (Figure $2 b$, blue data points vs. red data points at $\mathrm{SNR}=0.5$; see also Figure 2c, left).

To decompose these different effects of the pre-test tone sequence on choice and estimate the contributions of individual tone bursts, we fit a logistic model that included for each tone-burst position: 1) an additive bias term describing the degree to which choice was biased in the same (positive values) or opposite (negative values) direction as the frequency of that tone burst; and 2) an SNR-dependent, adaptation-like term describing the change in discriminability attributable to that tone burst, in which negative values corresponded to incongruent choices. These fits yielded, on average, a positive bias and a negative SNR-dependent adaptation-like component. Both the bias and adaptation-like terms had a strong recency effect in which the final 2-3 tone bursts just before the test tone made the strongest contributions to choice (Figure 2c, right). This bias + adaptation model was favored over both a bias-only model (mean $\Delta \mathrm{AIC}=2.22)$ and a model that did not include an effect of the tone bursts (mean $\triangle \mathrm{AIC}=56.85)$. A Bayesian 
181 random-effects analysis confirmed the bias + adaptation model as the most likely (protected

182 exceedance probability $=0.86$ ). This negative adaptation effect was unique to the stimulus-based

183 condition: adaptation-like terms estimated in the rule-based condition did not differ significantly

184 from 0 (sign test, all $p \mathrm{~s}>0.05$ ).

185 For the stimulus-based condition, we found similarly complex effects for average correct RTs

186 across subjects, which showed a main effect of congruence $(F(3,74.02)=27.76, p<0.0001)$ and

187 an $\operatorname{SNR} \times$ congruence interaction $(F(3,197.17)=11.58, p<0.0001)$. This interaction included a

188 tendency for faster/slower RTs when the last two pre-test tone bursts were congruent versus

189 incongruent with choice at the lowest/highest SNR (post-hoc test of interaction, $B=112.26$,

$\left.190 t(442.16)=5.06, p_{\text {corrected }}<0.0001\right)$. In summary, the subjects' choices were biased to be

191 congruent with the most-recently heard pre-test tone bursts at low SNRs but were biased to be

192 incongruent with those tone bursts at high SNRs. Together, these findings suggest the possibility

193 of separate computational mechanisms for the rule-based and stimulus-based effects, which we

194 examine next in more detail. 
a

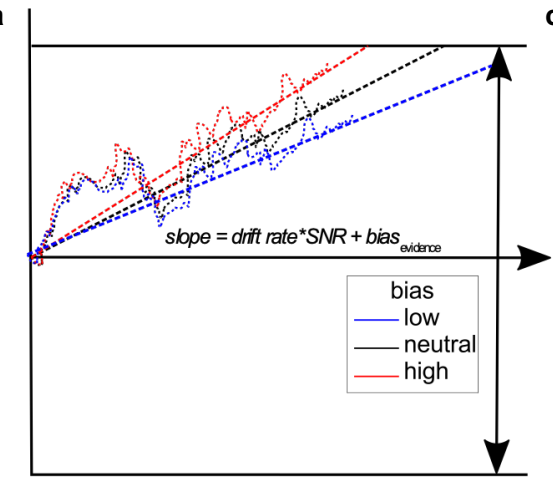

b
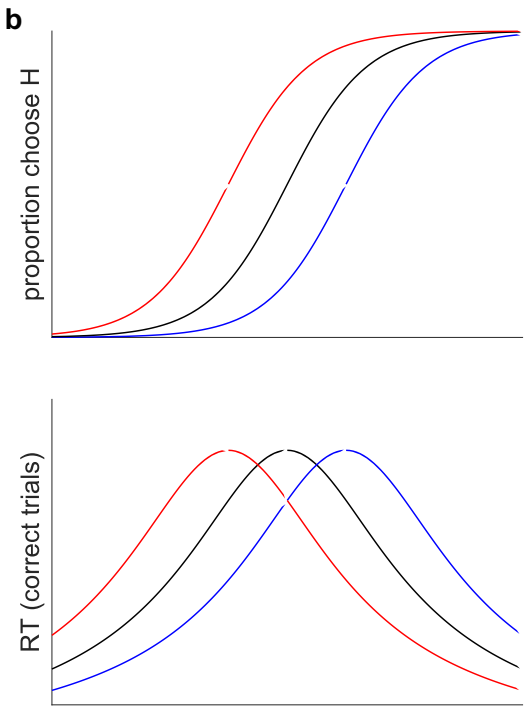

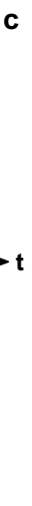

d
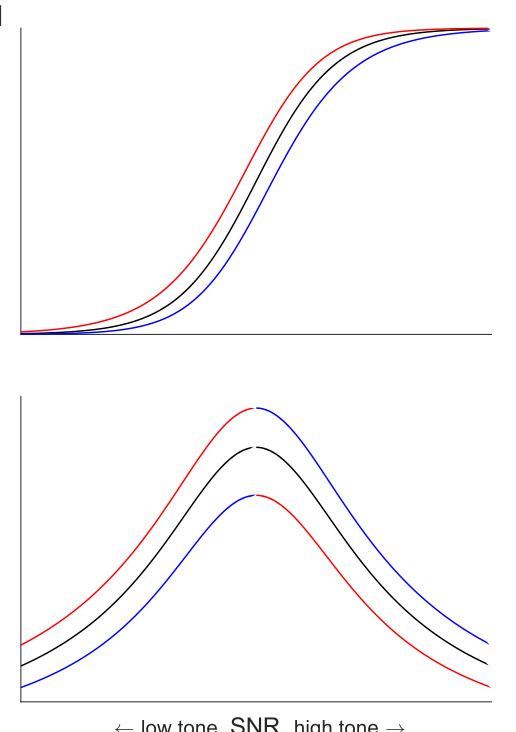

e

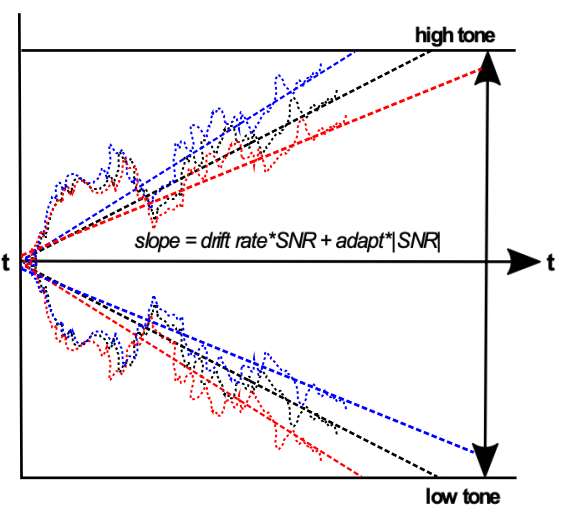

f
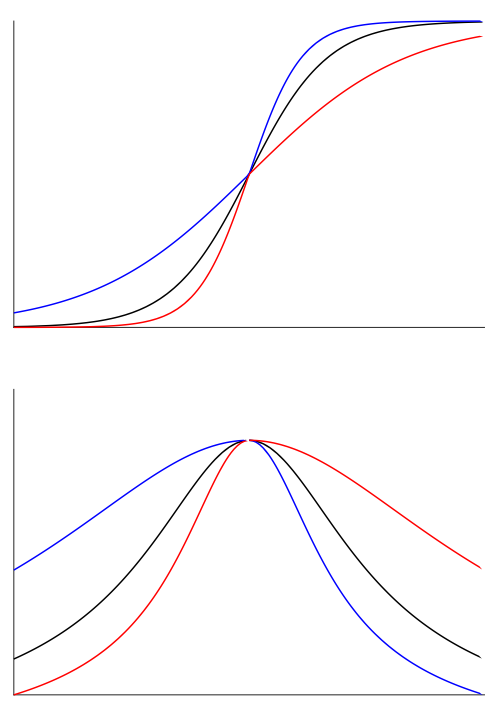

Figure 3. Simulated DDM bias terms (top) and their predicted, distinguishable effects on psychometric (choice; middle) and chronometric (RT; bottom) functions. a A bias implemented as an additive offset to the drift-rate term, which shifts the slope of the decision variable in the direction favored by the bias. b This evidence-accumulation bias causes lateral shifts in the psychometric and chronometric functions, corresponding to more and faster choices in the direction of the bias. $\mathbf{c}$ A bias implemented as a shift in the starting point of evidence accumulation toward the bound favored by the bias, implying that less evidence is needed to make a bias-congruent choice. $\mathbf{d}$ This starting-point bias causes smaller lateral shifts in the psychometric function than for the evidence-accumulation bias and vertical asymmetries in the chronometric function (see the discontinuity at SNR =0) such that RTs are faster (slower) for prior congruent (incongruent) choices. e An adaptation-like bias that affects the slope of the decision variable in a manner that varies with the absolute value of the test-tone SNR. $\mathbf{f}$ For the stimulus-based condition, this term, which is absent at $\mathrm{SNR}=0$ and increases with increasing 
$209|\mathrm{SNR}|$ of the test tone, shifts the slope of the decision variable away from the frequency of the

210 tone bursts heard prior to the test tone and thus causes more and faster choices to the frequency

211 opposite the most-recently heard pre-test tone bursts. a,c,e Dotted lines: example evolutions of

212 the decision variable for a single trial with a given bias (see legend). Dashed lines: average slope

213 of decision variable. $\mathrm{t}=$ time. In $\mathbf{c}, B=$ total bound height $/ 2$. b,d,f Simulated choice (top) and RT

214 (bottom) data from an analytical solution to the DDM (Palmer, Huk, \& Shadlen, 2005).

215 


\section{Rule- and stimulus-based biases were captured quantitatively by drift-diffusion model fits}

217 To better identify the computations responsible for these effects, we fit DDMs to the behavioral

218 data from each task condition (Shinn, Lam, \& Murray, 2020). Unlike the regression-based

219 approaches above, DDMs can jointly account for choice and RT data in a unified framework. In

220 the DDM, noisy evidence is accumulated to a pre-defined bound, which we modeled using five

221 free parameters: 1) drift rate, which influences the rate at which the test stimulus contributed to

222 evidence accumulation; 2) bound height, or the total evidence required to commit to a decision at

223 the beginning of the trial; 3) bound slope, which determined the rate of a linear "collapse" of the

224 bound over the course of a trial (i.e., a bound that decreases in height over time), to account for

225 potential urgency effects resulting from the time pressure to make the decision before the trial

226 was aborted after $2-3 \mathrm{~s} ; 4)$ non-decision time, or the portion of total reaction time that was not

227 determined by decision formation (e.g., sensorimotor processing); and 5) lapse rate, or error

228 rates for easily perceivable stimuli.

229 We added several terms to each model to account for bias effects (Figure 3). For the rule-based

230 condition, we used two additional free parameters per prior cue to account for biases in: 1) the

231 rate of accumulation, and 2) the starting point of the evidence-accumulation process. For the

232 stimulus-based condition, we used three additional parameters: 1) a bias in the rate of evidence

233 accumulation, 2) a bias in the starting point of the evidence-accumulation process, and 3) a

234 shared time constant that describes the rapid temporal decay in the influence of each pre-test tone

235 burst on the two biasing parameters (i.e., an exponentially weighted sum of biasing effects

236 caused by each preceding burst; Figure 2c, right). Positive/negative values for these bias terms

237 corresponded to more and faster choices toward/away from the dominant frequency of the pre-

238 test tone burst sequence (i.e., of the exponentially weighted sum). 
239 We introduced an additional mechanism to account for adaptation-like effects in the stimulus-

240 based condition by modulating the strength of evidence in an SNR-dependent fashion (Figure 3c,

$241 \mathrm{~d}$ ). Given the sharp decline in the effect of individual tone bursts (Figure 2c, right) and previous

242 work suggesting an exponential decay of auditory neural adaptation (Nelken, 2014; Pérez-

243 González, Hernández, Covey, \& Malmierca, 2012), we again modeled the adaptation-like effect

244 of the tone bursts as an exponentially weighted sum of the effects from each preceding tone burst

245 (with a shared time constant but separate, multiplicative weights for high- and low-frequency

246 tone bursts), which was then multiplied by the SNR of the test tone. Positive/negative weights

247 imply that repeated pre-test tone bursts caused more and faster choices toward/away from the

248 repeated tone frequency. Finally, we introduced an additional parameter to increase non-decision

249 time as a function of the absolute value of the stimulus-based bias, to capture the tendency for

250 faster RTs when the last two pre-test tone bursts were the same frequency (post-hoc test of

251 interaction, $B=68.83, t(72.07)=7.76, p<0.0001)$.

252 These models captured the patterns of bias and adaptation-like effects in the choice and RT data

253 (Figure 2a, b; solid lines). In the rule-based condition, the full model with cue-dependent bias

254 terms provided a better fit to the data than either the "collapsing-bound model" without cue-

255 dependent biases or a "base model" with neither cue-dependent biases nor a collapsing bound

256 (Table 1; protected exceedance probability =1). Similarly, in the stimulus-based condition, a

257 model with tone-burst-dependent biases outperformed models without these biases, and the full

258 model with both bias and SNR-dependent terms provided the strongest fit to the data (Table 1;

259 protected exceedance probability $=1$ ). Furthermore, the SNR-dependent terms were consistently

260 negative, confirming an adaptation-like effect (one-sample $t$-test; both $p \mathrm{~s}_{\text {corrected }}<0.0001$ ). 


\begin{tabular}{lllll} 
condition & model & $\begin{array}{l}\text { number of } \\
\text { parameters }\end{array}$ & mean AIC & $\begin{array}{l}\text { protected exceedance } \\
\text { probability }\end{array}$ \\
\hline rule-based & base & 6 & -66.00 & 0 \\
& $\begin{array}{l}\text { collapsing bound } \\
\text { full }\end{array}$ & 7 & -149.82 & 0 \\
stimulus-based & 11 & -267.28 & 1 \\
& base & 6 & 211.52 & 0 \\
& $\begin{array}{l}\text { collapsing bound } \\
\text { collapsing bound }+\end{array}$ & 7 & 133.29 & 0 \\
& $\begin{array}{l}\text { stimulus-based bias } \\
\text { full }\end{array}$ & 11 & 76.85 & 0 \\
& 14 & 52.02 & 1 \\
\hline
\end{tabular}

262 Table 1. DDM model-fit comparisons. In both conditions, the full model was favored over

263 reduced models, with the lowest AIC and a protected exceedance probability $=1$. All models

264 included evidence-acculumulation and starting-point bias terms to account for idiosyncratic

265 biases, even when they did not include cue-dependent biases. See Methods for full

266 implementation details. 
a

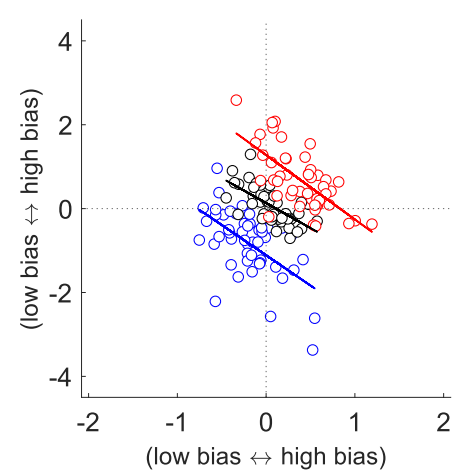

C

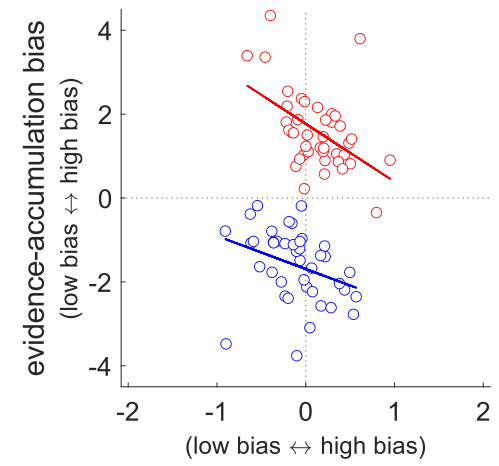

e

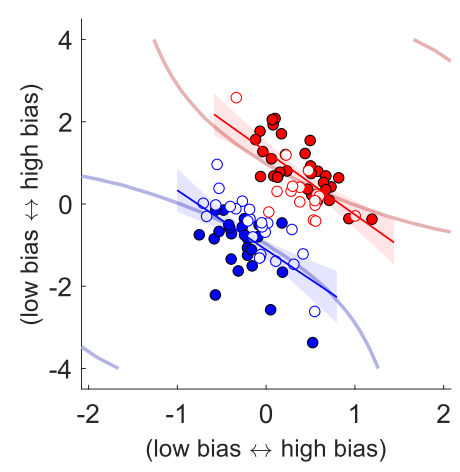

b

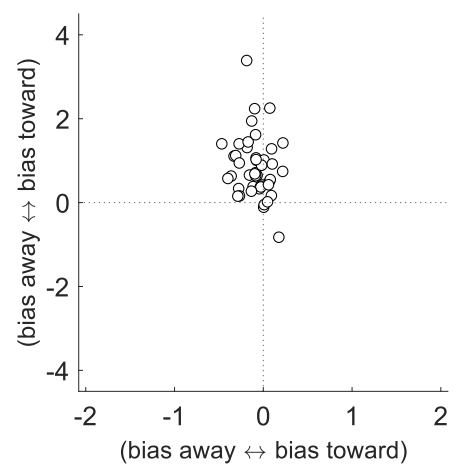

d

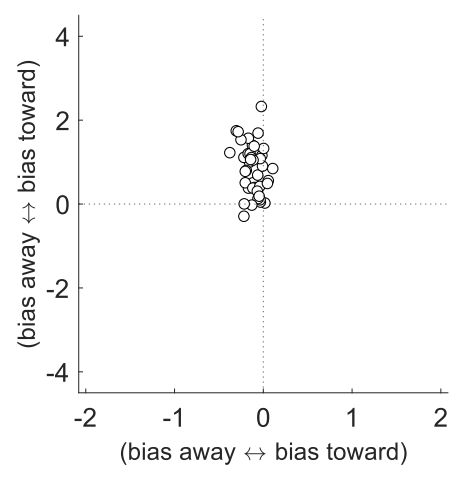

$\mathbf{f}$

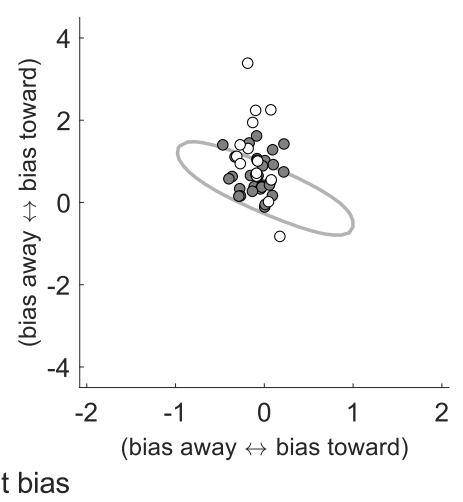

Figure 4. Different computational signatures of rule- and stimulus-based biases. Each panel

270 shows best-fitting evidence-accumulation (ordinate) and starting-point biases, plotted per subject

271 (points). a Data from the rule-based condition, for each of the three prior cues (blue: low, black:

272 neutral; red: high). Lines are linear fits $(p<0.05)$. b Data from in the stimulus-based condition

273 (no linear relationship). c Data from the rule-based mixed condition, plotted as in a. d Data from

274 the stimulus-based condition, plotted as in $\mathbf{b}$ (no linear relationship). $\mathbf{c}$ and $\mathbf{d}$ show results for the

275 mixed-condition 1, which included block-level prior cues; results were similar in mixed-

276 condition 2, which had trial-wise priors. e Data from a (the low- and high-prior conditions)

277 plotted with respect to optimal performance contours from the rule-based condition. The average $27897 \%$ contours (curved lines) demarcate the area of the parameter space in which choice accuracy 
279 is at least $97 \%$ of the maximum possible accuracy. Accuracy was predicted by the DDM applied

280 to the actual task sequences. Closed/open circles indicate that the data fell within/outside of the

281 subject-specific 97\% contour. Lines are linear fits as in a, and shaded regions are 95\%

282 confidence intervals. f Same as e, but for the stimulus-based condition. The $97 \%$ contour is for

283 the context of two low-tone bursts before the test tone (similar results were obtained for two

284 high-tone bursts but not shown).

285 


\section{Rule- and stimulus-based biases exhibited distinct computational signatures}

287 The rule- and stimulus-based bias effects had distinct computational signatures. For the rule-

288 based condition, subjects tended to use both starting-point and evidence-accumulation biases in

289 the direction of the higher-probability alternative (Table 2, light green rows; one sample $t$-test, all

$\left.290 p s_{\text {corrected }}<0.0001\right)$. Across subjects, these two kinds of computational biases were negatively

291 correlated with one another: subjects who tended to use stronger starting-point biases had weaker

292 evidence-accumulation biases and vice versa. This negative relationship was evident within all

293 three prior cue conditions (Figure 4a; low: Spearman's $\rho=-0.49, p_{\text {corrected }}=0.0003$; neutral: $\rho=$

$-0.72, p_{\text {corrected }}<0.0001$; high: $\left.\rho=-0.62, p_{\text {corrected }}<0.0001\right)$.

\begin{tabular}{|c|c|c|c|c|c|c|c|c|c|}
\hline \multirow{2}{*}{$\begin{array}{l}\text { condition } \\
\text { parameter }\end{array}$} & \multicolumn{2}{|c|}{ rule-based } & \multicolumn{2}{|c|}{$\begin{array}{c}\text { stimulus- } \\
\text { based }\end{array}$} & \multicolumn{2}{|c|}{ mixed 1} & \multicolumn{2}{|c|}{ mixed 2} & \multirow[b]{2}{*}{ description } \\
\hline & mean & SD & mean & SD & mean & SD & mean & SD & \\
\hline VSNR & 6.51 & 3.36 & 4.97 & 2.55 & 4.40 & 2.34 & 5.46 & 3.21 & drift rate \\
\hline B & 0.92 & 0.26 & 1.02 & 0.27 & 1.06 & 0.21 & 0.93 & 0.17 & bound height (total bound height $=2 \mathrm{~B}$ ) \\
\hline$t_{B}$ & 0.92 & 0.48 & 0.80 & 0.33 & 1.01 & 0.38 & 0.87 & 0.45 & bound slope \\
\hline ndt $_{0}$ & 0.27 & 0.08 & 0.46 & 0.07 & 0.38 & 0.05 & 0.07 & 0.07 & non-decision time $(\mathrm{s})$ \\
\hline ndt $_{\text {Bias }}$ & $*$ & $*$ & -0.12 & 0.29 & -0.12 & 0.32 & -0.17 & 0.29 & $\begin{array}{l}\text { modulation of non-decision time by absolute } \\
\text { stimulus-based bias }\end{array}$ \\
\hline$\lambda$ & 0.02 & 0.01 & 0.02 & 0.02 & 0.02 & 0.01 & 0.02 & 0.01 & lapse rate \\
\hline VLow & -0.83 & 0.79 & $*$ & $*$ & -1.60 & 0.85 & -1.36 & 0.91 & Evience-accumulation bias for low prior \\
\hline $\mathrm{V}_{0}$ & 0.07 & 0.41 & -0.20 & 0.62 & $*$ & $*$ & -0.19 & 0.61 & $\begin{array}{l}\text { Evidence-accumulation bias for neutral prior } \\
\text { (idiosyncratic) }\end{array}$ \\
\hline VHigh & 0.67 & 0.71 & $*$ & $*$ & 1.59 & 0.93 & 1.19 & 1.04 & Evidence-accumulation bias for high prior \\
\hline VBias & $*$ & $*$ & 0.84 & 0.73 & 0.83 & 0.60 & 0.80 & 0.49 & stimulus-based evidence-accumulation bias \\
\hline$\tau_{\text {Bias }}^{-1}$ & $*$ & $*$ & 4.99 & 4.89 & 6.82 & 5.24 & 7.80 & 5.11 & time constant of stimulus-based bias \\
\hline vaLow & $*$ & $*$ & -2.31 & 1.81 & -2.05 & 1.83 & -1.97 & 1.37 & adaptation weight, low tones \\
\hline vaHigh & $*$ & $*$ & -1.40 & 1.75 & -1.53 & 1.67 & -1.68 & 1.22 & adaptation weight, high tones \\
\hline$\tau_{\mathrm{va}}^{-1}$ & $*$ & $*$ & 7.04 & 6.25 & 9.84 & 5.45 & 8.74 & 5.97 & time constant of adaptation \\
\hline ZLow & -0.20 & 0.31 & $*$ & $*$ & -0.12 & 0.36 & 0.00 & 0.30 & starting point bias for low prior \\
\hline $\mathrm{Z}_{0}$ & 0.06 & 0.22 & -0.03 & 0.19 & $*$ & $*$ & 0.02 & 0.24 & starting point bias for neutral prior (idiosyncratic) \\
\hline$Z_{\text {High }}$ & 0.39 & 0.31 & $*$ & $*$ & 0.13 & 0.33 & 0.03 & 0.35 & starting point bias for high prior \\
\hline ZBias & $*$ & $*$ & -0.09 & 0.16 & -0.11 & 0.10 & -0.03 & 0.17 & stimulus-based starting-point bias \\
\hline
\end{tabular}

Table 2. Mean and standard deviation (SD) of best-fitting paramaters across subjects.

297 Green rows: rule-based bias parameters; yellow rows: stimulus-based bias parameters; *

298 parameter not present in that condition. See Methods for a fuller description of the parameters. 
For the stimulus-based condition, subjects also tended to use a combination of starting-point and evidence-accumulation biases but with a different pattern than for the rule-based condition (Table 2, light yellow rows). Specifically, the evidence-accumulation bias tended to be positive (i.e., toward the frequency of the most recent tone bursts; one-sample $t$-test, $p<0.0001$ ). In contrast, the starting-point bias tended to be in the opposite direction, away from the bound for the frequency of the most recent tone bursts $(p=0.0007)$. Unlike for the rule-based DDM fits, we could not identify any correlation between best-fitting values of evidence-accumulation and starting-point biases across subjects (Figure 4b; Spearman's $\rho=-0.20, p=0.19$ ). In the mixedcondition trials, we confirmed that this differential pattern of correlations was also present and thus was not simply an idiosyncratic quirk of either the rule- or stimulus-based condition tested individually (rule-based: Figure $4 \mathrm{c}$, all $p \mathrm{~s}_{\text {corrected }}<0.005$; stimulus-based: Figure $4 \mathrm{~d}$, all $p \mathrm{~s}>$ $0.05)$.

The negative relationship between evidence-accumulation and starting-point biases in the rulebased conditions is similar to that found for monkeys performing a reward-biased visual-decision task (Doi, Fan, Gold, \& Ding, 2020; Fan, Gold, \& Ding, 2018). That reward-driven relationship was interpreted in terms of coupled, goal-directed (top-down) adjustments to the decision process that improved performance. Specifically, monkeys made adjustments in evidenceaccumulation and starting-point biases that were sensitive to session- and monkey-specific variations in perceptual sensitivity and contextual factors. These adjustments maintained nearoptimal or "good enough" (satisficing) performance, possibly by using a gradient-ascent-like learning process to rapidly adjust the biases until reaching a performance plateau. Critically, the contours of this performance plateau showed a "tilt" in the space of evidence-accumulation and starting-point biases, such that negatively correlated values of these biases traced out iso- 
323 performance contours. We reasoned that a similar sensitivity to performance-satisficing

324 combinations of biases could plausibly underlie the correlation between the evidence-

325 accumulation and starting-point biases in our rule-based condition.

326 To assess this possibility, we used the DDM to predict, for each subject, the average choice

327 accuracy that could be achieved across a range of evidence-accumulation and starting-point bias

328 parameters, given the subject's other fit parameters and the task context. For the rule-based

329 condition, task context was determined by the prior cue. For the stimulus-based condition, task

330 context was defined with respect to two high or two low pre-test tone bursts, which had the

331 strongest effects on behavior in that condition. To facilitate comparisons, we normalized

332 predicted accuracy for each subject and condition to the maximum value achieved via

333 simulations using a wide range of evidence-accumulation and starting-point bias parameter

334 values.

335 For the rule-based condition, the subjects' combinations of evidence-accumulation and starting-

336 point biases tended to yield nearly optimal performance (low: median proportion maximum

337 performance $=0.97$, range $=[0.76-1.00]$; neutral: 1.00 [0.97-1.00]; high: 0.98 [0.86-1.00]).

338 Furthermore, the location and tilt of the across-subjects distribution of biases appeared

339 qualitatively to respect the tilt of the plateau of the performance function (Figure 4d), suggesting

340 that sensitivity to this plateau was a potential cause of the correlation between the biases. For the

341 stimulus-based condition, the subjects' biases also yielded nearly optimal performance (LL:

342 median proportion max performance $=0.99$, range $=[0.62-1.00] ; \mathrm{HH}: 0.99$ [0.80-1.00]),

343 reflecting the fact that some degree of positive bias was needed to offset performance decrements

344 induced by the adaptation-like effect. However, the subjects' biases for the stimulus-based

345 condition did not follow the tilt of the plateau of the performance function (Figure 4e). 
346 To confirm this pattern of results, we used a mixed-effects model to compare the slopes of the

347 linear fit that predicted the starting-point bias from the evidence-accumulation bias between the

348 different contexts. We could not identify any difference in the slopes of the high versus low

349 biases within the rule-based condition $(B=-0.03, t(126.00)=-0.14, p>0.05)$. In contrast, there

350 was a significant difference in the slope of the biases between the rule-based and stimulus-based

351 conditions $(B=0.45, t(126.00)=2.80, p=0.006)$. Together, these findings are consistent with

352 the idea that the participant's choices in the stimulus-based condition were produced by a

353 different underlying mechanism than the coupled (tilted) changes that mediated choices in the

354 rule-based condition.

355 Consistent with this idea of different mechanisms, we could not find any evidence that individual 356 subjects made consistent use of starting-point and/or evidence-accumulation biases across the 357 rule-based and stimulus-based conditions. Specifically, there was no correlation between best-

358 fitting values of each bias term computed per subject when comparing across the two conditions

359 ( $p$ s $>0.05$ for both starting-point and evidence-accumulation biases). When rule-based and

360 stimulus-based biases were used at the same time in the mixed conditions, we also could not

361 identify a correlation between the bias terms (all ps $>0.05$; note that parameter ranges were

362 similar for the single and mixed blocks, suggesting that the subjects used the biases in a roughly

363 comparable, although not identical, way in the two block types; Table 2). Together, these results

364 suggest that rule-based and stimulus-based biases rely on separable computational processes,

365 potentially reflecting differences in top-down and bottom-up sources of expectations. 
a

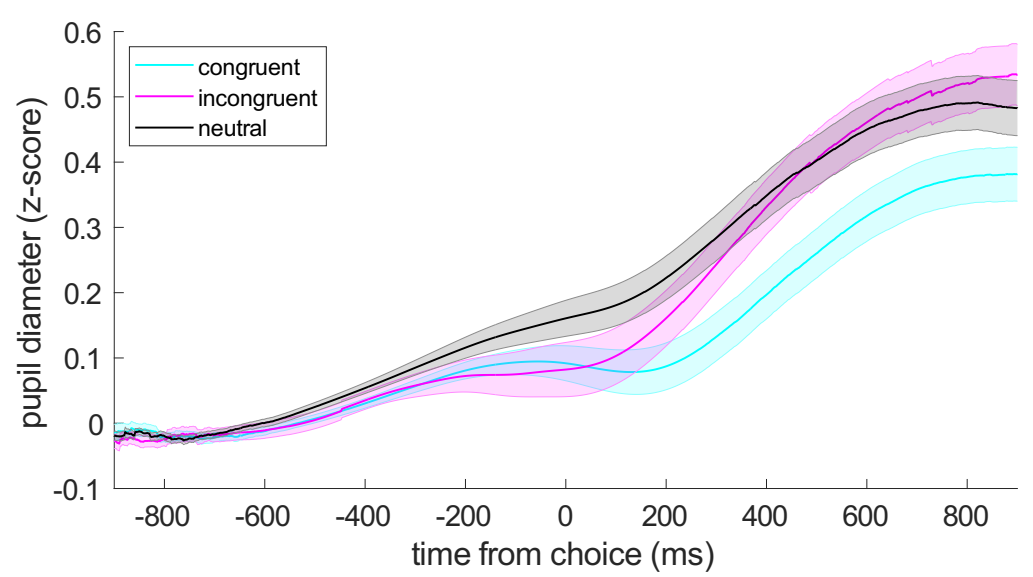

b

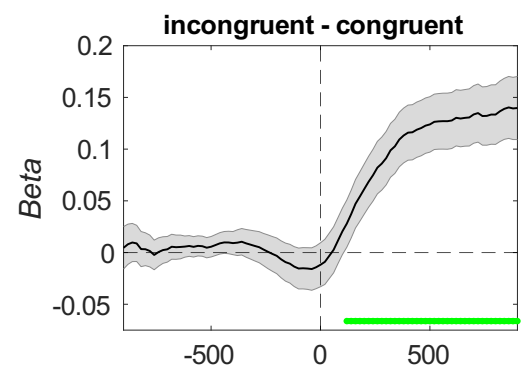

C

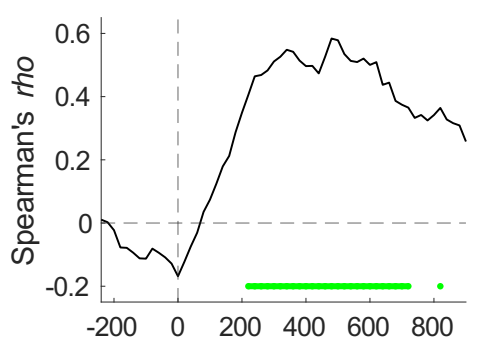

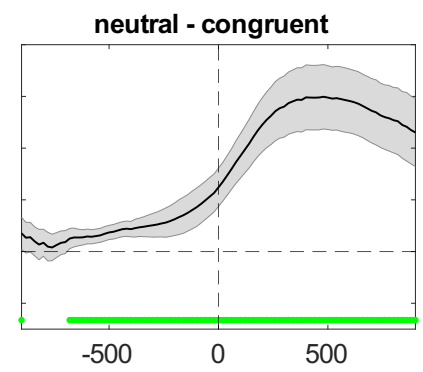
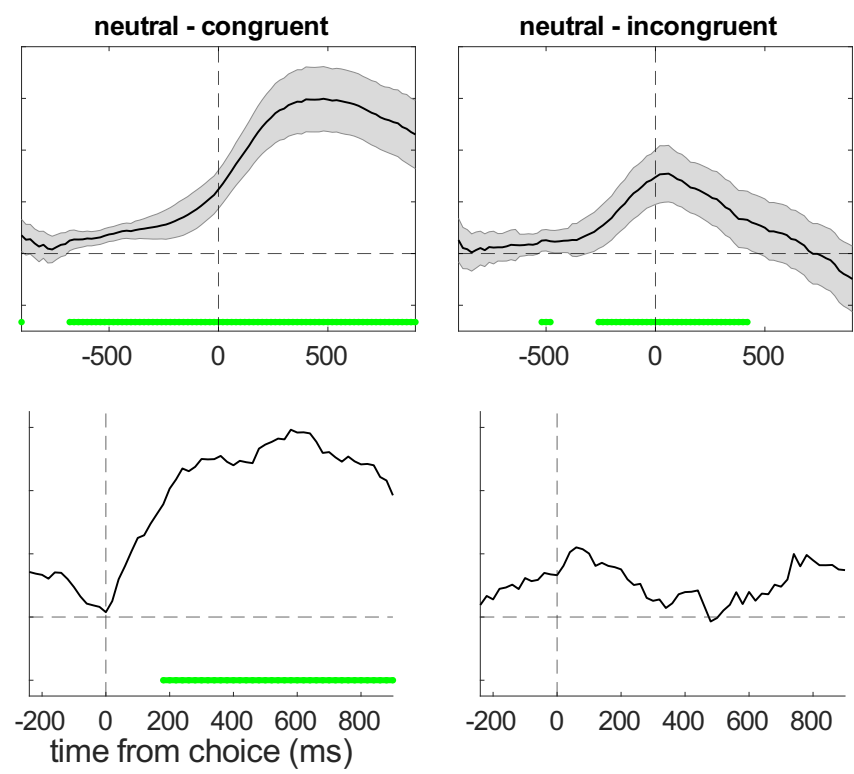

Figure 5. Rule-based cues strongly modulated pupil-linked arousal. a Pupil diameter on correct trials, aligned to choice and plotted as a function of the congruence between the choice and the prior. Lines and error bars are means and SEM across all trials and subjects. b Regression coefficients (beta values) from a linear mixed-effects model as a function of different pupil contrasts (see labels above each panel). Error bars are 95\% confidence intervals of the parameter estimates. c Across-subject Spearman correlation between: 1) the degree to which each subject was biased by the prior cues, estimated from a logistic regression model of choice behavior; and 2) each subject's pupil contrast from a linear regression model. Values in $\mathbf{b}$ and $\mathbf{c}$ were computed in 20 ms-wide time bins. Green lines indicate time bins for which $p_{\text {corrected }}<0.05$, FDR-corrected across time and contrast. Figure 5 - Supplement 1 presents the same analyses as in $\mathbf{a}$ and $\mathbf{b}$ for incorrect trials. 


\section{Rule- and stimulus-based biases exhibited distinct physiological signatures}

380

381

382

Because pupil dilation is associated with behavioral decision biases (de Gee et al., 2017, 2014, 2020; Krishnamurthy et al., 2017; Urai et al., 2017), we tested whether and how pupil size was modulated by our rule-based and stimulus-based task conditions. In particular, we tested whether these modulations could help distinguish different forms of biases across individuals and task conditions. If pupil-linked arousal facilitates overcoming biases, then we would expect larger evoked pupil responses when comparing incongruent to congruent trials: responding correctly on incongruent trials requires overcoming bias. Furthermore, if the rule- and stimulus-based biases depend on overlapping neurophysiological mechanisms, then we would expect similar profiles of pupil responses for both conditions. In contrast, if pupil-linked arousal for our task is more sensitive to top-down than to bottom-up influences, as has been reported for other tasks (Filipowicz et al., 2020), then we would expect more strongly modulated pupil responses in the rule-based than in the stimulus-based condition.

In the rule-based condition, the choice-locked evoked pupil response was modulated strongly by congruence (Figure 5a, b). Specifically, the pupil response was larger for incongruent relative to congruent choices, an effect that emerged shortly after choice (because the effects were similar but weaker when aligned to stimulus versus choice onset, the figures and analyses focus on choice-aligned effects). We found a similar effect on incorrect trials (Figure 5 - Supplement 1), which implies that these pupil modulations were more closely related to the congruency between the prior and the choice than to the congruency between the prior and the stimulus. Pupil diameter was also modulated on both congruent and incongruent trials relative to neutral trials, with larger dilations on neutral trials starting before stimulus onset that likely reflected higher uncertainty on those trials (i.e., a less-informative prior; Figure 5a,b). In the mixed condition, 
402 similar rule-based modulations were also evident and thus were robust to the presence or absence

403 of stimulus-based biases (mixed-effects models comparing average pupil responses 220-720 ms

404 post-choice for pairs of prior cues, all $p \mathrm{~s}_{\text {corrected }}<0.03$, except for the no prior - incongruent

405 contrast in mixed block $2, p>0.05)$.

406 The magnitude of these rule-based pupil modulations was correlated with the magnitude of

407 behavioral biases across subjects. Specifically, we measured the Spearman correlation coefficient

408 between the magnitude of choice bias derived from the logistic fits to each subject's choice data

409 (high-frequency cue bias minus low-frequency cue bias) and pupil contrast ( $\beta$ value). We found

410 positive correlations $\sim 200-800 \mathrm{~ms}$ post-choice for both the congruent-incongruent and neutral-

411 congruent contrasts. In other words, a greater reliance on the prior cues corresponded to more

412 differentiated arousal responses between trials when the cue was or was not congruent with the

413 choice. These results complement earlier findings that pupil responses can reflect the magnitude

414 of (inappropriate) choice biases, with larger pupil responses typically corresponding to a

415 reduction in bias (de Gee et al., 2017, 2014, 2020; Krishnamurthy et al., 2017). Taken together

416 with our results, these findings suggest that our subjects, particularly those who were highly

417 sensitive to the prior cues, needed to mobilize additional resources to overcome the prior or make

418 decisions in the absence of an informative prior. 
a

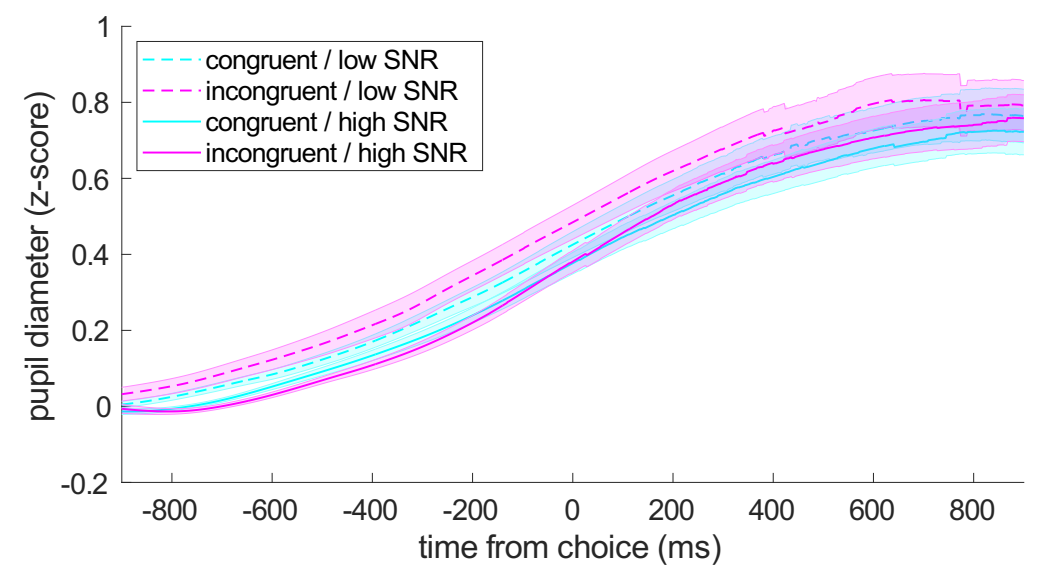

b
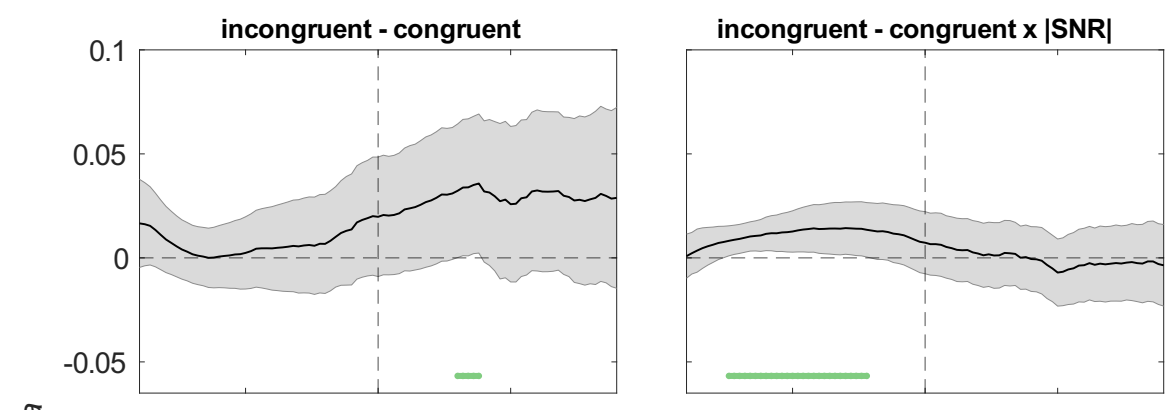

$\frac{\pi}{\mathbb{\infty}}$
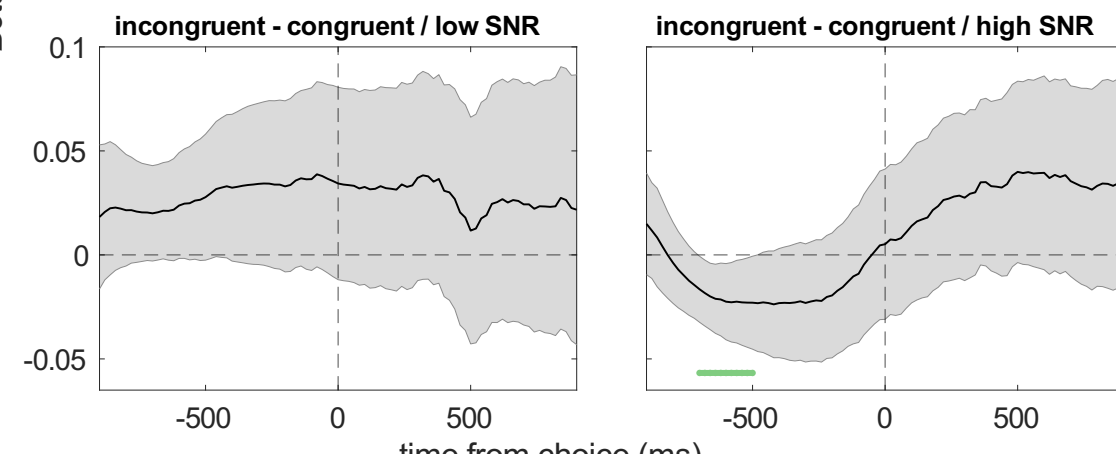

Figure 6. Stimulus-based cues weakly modulated pupil-linked arousal. a Pupil diameter on correct trials, aligned to choice and plotted as a function of the congruence of the choice and the most-recently heard pre-test tone bursts, separated by SNR. We only used pre-test sequences ending in two of the same tone (i.e., LL, HH) and at the lowest or highest test SNR for this analysis. Lines and error bars are means and SEM across all trials and subjects. b Regression 425 coefficients (beta values) from a linear mixed-effects model for different pupil contrasts. Error bars are $95 \%$ confidence intervals of the parameter estimates. Values were computed in 20 mswide time bins. Dark green lines indicate time bins for which $p_{\text {uncorrected }}<0.05$. 
429 In contrast, evoked pupil diameter in the stimulus-based condition displayed a very different

430 pattern of results. Because the subjects' behavior in this condition was dependent on congruence

431 and SNR, we analyzed pupil diameter as a function of the interaction between: 1) the congruence

432 of choice with the last two pre-test tone bursts, and 2) SNR. We focused on trials in which both

433 tone bursts were the same and for only the lowest or highest test SNR values, to increase our

434 chances of finding effects. However, unlike for the rule-based condition, we did not identify any

435 effects that survived correction for multiple comparisons (Figure 6). Further, we found a

436 significant congruence $\times$ condition interaction (mixed-effects model comparing average pupil

437 responses $0-1000 \mathrm{~ms}$ post-choice, $B=0.09, t(38.36)=5.65, p<0.0001)$, which confirmed that

438 the arousal response was stronger in the rule-based condition than in the stimulus-based

439 condition. Although we could not identify any sign of stimulus-based effect in the post-choice

440 period, we found a congruence $\times$ SNR interaction at an uncorrected $p<0.05$ from $740-220 \mathrm{~ms}$

441 before choice. We also reanalyzed the rule-based condition to look for a similar

442 congruence $\times$ SNR interaction and could not identify any effect (all $p \mathrm{~s}>0.05$ ).

443 In summary, pupil-linked arousal strongly differentiated between the rule- and stimulus-based

444 biases in the locus, strength, and timing of the effect. In the rule-based condition, pupil diameter

445 following choice was robustly indicative of the congruence of the choice and the prior cue in a

446 manner that reflected individual behavioral biases. In the stimulus-based condition, pupil

447 diameter preceding choice only weakly reflected the SNR-dependent choice congruence effects. 


\section{Discussion}

450 This work was motivated by the realization that, despite an abundance of studies on the

451 importance of expectations in auditory processing, we lack a basic understanding of their effects

452 on perception. We focused on how human auditory perceptual decisions are influenced by two

453 different sources of expectations, one from instructed rules that indicated the probability of the

454 upcoming test tone and the other from stimulus regularities akin to those used in oddball tasks.

455 Rule-based cues consistently biased behavioral choices and RTs in favor of the more probable

456 alternative. In contrast, stimulus-based cues elicited different biases that depended on the test-

457 tone SNR. We leveraged model fits and measures of pupil-linked arousal to show that the rule-

458 based and stimulus-based behavioral biases have distinct computational and physiological

459 signatures: we found coordinated computational adjustments and strong pupil modulations in the

460 rule-based condition but not in the stimulus-based condition.

461 Our results extend previous findings in three fundamental ways. First, we decomposed the

462 effects of stimulus regularities on auditory perception into an adaptation-like effect on stimulus

463 sensitivity and a decision bias. This decomposition allowed us to directly compare decision

464 biases produced by rule-based versus stimulus-based cues. Second, we found that rule-based and

465 stimulus-based biases have distinct computational signatures. In particular, the effects on the

466 decision variable (i.e., evidence-accumulation bias in the DDM) and the decision rule (i.e.,

467 starting-point bias in the DDM) are coupled for rule-based biases but not for stimulus-based

468 biases. Third, we showed that rule-based and stimulus-based biases also have distinct

469 physiological signatures: pupil-linked arousal is modulated by rule-based biases but not by

470 stimulus-based biases. As detailed below, we interpret these results in terms of two potentially

471 distinct mechanisms for incorporating prior expectations into auditory perceptual decisions, one 
472 using top-down processing for rule-based cues and the other using bottom-up processing for

473 stimulus-based cues.

474 The rule-based cues in our task were visual, not auditory, and thus were processed separately

475 from the incoming auditory stream and then incorporated into the auditory decision. Several of

476 our findings imply that this process involved cognitively driven, top-down mechanisms and not

477 lower-level interactions between the visual and auditory stimuli. Specifically, we modeled the

478 rule-based decision biases in terms of computational components of a normative, DDM-based

479 decision process. These kinds of computationally defined biases, which have been studied

480 extensively for visual decision-making (Dunovan et al., 2014; Hanks et al., 2011; Kelly et al.,

481 2020; Mulder et al., 2012; Ratcliff \& McKoon, 2008; Seriès \& Seitz, 2013) but only sparsely for

482 auditory decision-making (de Gee et al., 2020; Gifford et al., 2014), have been ascribed to top-

483 down influences (Summerfield \& de Lange, 2014). Our results further support this idea by

484 showing auditory decision biases can occur flexibly from trial-to-trial, depending on the

485 relationship between the cue and the test stimulus. Moreover, these biases involve computational

486 components that are coordinated with each other, which we previously showed is consistent with

487 a cognitive-based (i.e., not purely stimulus-driven) learning process that can allow individual

488 decision-makers to achieve nearly optimal performance (Fan et al., 2018).

489 Likewise, the rule-based biases that we measured were accompanied by modulations of pupil

490 size that, like for related findings, appear to be cognitively driven (Joshi \& Gold, 2020).

491 Specifically, we found transient pupil increases in response to choices that were incongruent with

492 the prior cue on that trial. These pupil modulations were similar to those that have been reported

493 for other tasks involving decision biases (de Gee et al., 2017, 2014, 2020; Krishnamurthy et al.,

494 2017; Urai et al., 2017), violations of learned expectations (Filipowicz et al., 2020), and certain 
task-relevant but not task-irrelevant stimulus changes (Zhao et al., 2019), all of which were interpreted in terms of arousal-linked cognitive influences on perception and/or decision processing. Our work complements and extends these findings by showing that the rule-based effects on task behavior and pupil size can be adjusted from trial-to-trial, based on the current version of the cue and its relationship (congruence) with the test stimulus, further supporting the idea that they are driven by the kinds of flexible information processing associated with topdown control.

Together, these results are consistent with the idea that arousal-linked processes actively monitor and adjust decision-making using relevant predictive cues. These adjustments likely arise from multiple neuromodulatory systems, principally the locus-coeruleus (LC) norepinephrine system and basal forebrain cholinergic system (Joshi \& Gold, 2020; Joshi, Li, Kalwani, \& Gold, 2016; Reimer et al., 2016), that can affect either bottom-up or top-down information processing in the brain under different conditions. For example, relatively slow fluctuations in baseline or "tonic" arousal levels have been linked to modulation of sensory neurons (Heller, Schwartz, Saderi, \& David, 2020; Lin, Asinof, Edwards, \& Isaacson, 2019; McGinley, David, \& McCormick, 2015; McGinley, Vinck, et al., 2015; Reimer et al., 2014; Schwartz, Buran, \& David, 2020; Vinck, Batista-Brito, Knoblich, \& Cardin, 2015) and changes in perceptual sensitivity in animal models and human subjects (Gelbard-Sagiv, Magidov, Sharon, Hendler, \& Nir, 2018; McGinley, David, et al., 2015; Waschke, Tune, \& Obleser, 2019), suggesting bottom-up effects. In contrast, relatively fast, event-driven "phasic" changes in arousal have shown inconsistent relationships to perceptual sensitivity in human subjects (de Gee et al., 2017, 2020). Rather, these phasic changes (like what we measured) are more strongly associated with behaviorally relevant decision- and response-related processes in both humans and animal models (Aston-Jones \& Cohen, 2005; de 
518 Gee et al., 2014; Einhäuser, Koch, \& Carter, 2010; Kalwani, Joshi, \& Gold, 2014), particularly

519 those requiring cognitive or behavioral shifts (Bouret \& Sara, 2005), which is suggestive of top-

520 down effects. Our results support this interpretation: the pupil response was modulated by the

521 congruency between the cue and the subject's choice, not the cue and the tone identity,

522 consistent with arousal-linked adjustment of top-down decision biases rather than bottom-up

523 sensory processing. This interpretation accords with a previous visual decision-making study

524 showing that pupil-linked arousal modulates decision biases in frontoparietal regions encoding

525 choice, without any effect on visual cortex (de Gee et al., 2017). Further work is needed to

526 identify whether similar neural dissociations exist in auditory decision-making.

527 In contrast, the stimulus-based cues appeared to engage a different set of mechanisms that were 528 more consistent with contributions from bottom-up processing. In particular, the stimulus-based 529 cues elicited behavioral biases that included both "attractive" effects (i.e., biases towards 530 previous stimuli) and "repulsive" effects (i.e., biases away from previous stimuli). Indeed, both

531 of these effects have been identified in previous studies of auditory perception (Alais et al., 2015;

532 Chambers et al., 2017; Chambers \& Pressnitzer, 2014; Dahmen, Keating, Nodal, Schulz, \&

533 King, 2010; Giangrand, Tuller, \& Kelso, 2003; Holt \& Lotto, 2008; Shu et al., 1993; Snyder,

534 Carter, Hannon, \& Alain, 2009) and auditory short-term memory (Lieder et al., 2019). However, 535 most of these prior studies reported consistently attractive or repulsive effects (cf. Snyder et al.,

5362009 for opposing effects of prior stimuli and prior perception) but not both, leaving us without a

537 principled explanation for the conditions under which one or the other effect should occur. To

538 our knowledge, we are the first to report that the direction of bias depended strongly on SNR,

539 with attractive decision biases predominating for low-test-tone-SNR stimuli and repulsive, 540 adaptation-like mechanisms that reduced sensitivity to repeated stimuli predominating for high- 
test-tone-SNR stimuli. The stimulus-based biases were unrelated to rule-based biases and did not

542 show any learning-related coordination within subjects. Moreover, these stimulus-based effects

543 had little relationship to pupil size, unlike the rule-based effects. Thus, these effects did not

544 appear to engage the top-down, arousal-linked cognitive mechanisms used to generate rule-based

545 biases but instead were induced by the tone sequence itself.

546 These stimulus-based effects were likely driven, in part, by mechanisms of sensory adaptation.

547 These mechanisms affect neural responses throughout the auditory system and generally involve

548 reduced responses to repeated presentation of the same stimuli and potentiated responses to

549 novel (deviant) stimuli (Carbajal \& Malmierca, 2018; Näätänen et al., 2007; Nelken, 2014;

550 Sussman, 2007). There is debate over whether these phenomena reflect predictions or whether

551 they are indicative of traditionally construed bottom-up stimulus-specific adaptation, with

552 evidence suggesting that both processes may contribute (Carbajal \& Malmierca, 2018; Heilbron

553 \& Chait, 2018; Lesicko et al., 2021; Nelken, 2014; Parras et al., 2017; Symonds et al., 2017;

554 Todorovic \& de Lange, 2012; Todorovic et al., 2011). Both interpretations are consistent with

555 our findings, in that the attractive effects could reflect predictions whereas the repulsive effects

556 are more consistent with adaptation.

557 A possible reason for the coexistence of the opposing stimulus-based effects is to allow the brain

558 to navigate the tradeoff between stable integration and change detection as a function of the

559 quality of sensory evidence (Glaze, Kable, \& Gold, 2015; Schwiedrzik et al., 2014). When SNR

560 is low, the evidence is unreliable and thus prior expectations are most useful. In contrast, when

561 SNR is high and prior expectations are not as useful, other aspects of stimulus processing can be

562 prioritized, such as increasing sensitivity to deviations from stimulus regularities. Past normative

563 models of evidence accumulation in changing environments have accounted for human subjects' 
564 sensitivity to change via a volatility-dependent leak on the prior accumulated evidence (Glaze et

565 al., 2015). Future work should investigate whether adaptation-induced changes in the encoding

566 of sensory evidence could also account for subject behavior (Fritsche, Spaak, \& de Lange, 2020;

567 Schwiedrzik et al., 2014; Stocker \& Simoncelli, 2005; Wei \& Stocker, 2015), and whether

568 environmental volatility might alter the balance between attractive and repulsive effects in

569 auditory decision making.

570 In sum, the results of this study established new methods for understanding how stimulus- and

571 rule-based information shape auditory decisions, jointly within a DDM framework. Future work

572 should leverage this framework to identify the neural substrates of these effects and should

573 further establish the stimulus dimensions that govern the balance between attractive and

574 repulsive effects, which has implications for understanding how the brain balances reliance on

575 prior information with sensitivity to change.

576 
Materials and methods

578 Fifty human subjects participated in the study (19 male, 25 female, 6 N/A; median age: 25 yrs,

579 range 18-60). Informed consent was obtained in accordance with the University of Pennsylvania

$580 \quad$ IRB.

\section{Behavioral Task}

582 Each subject was seated and faced an LCD computer screen (iMac), which sat on a table in a 583 darkened room. The subject listened to auditory stimuli via earphones (Etymotic MC5), while a 584 chin rest stabilized their head for eye tracking. The subject reported whether a "test" tone burst 585 (250 or $2000 \mathrm{~Hz}$; 300-ms duration; 10-ms $\cos ^{2}$ ramp) was "low frequency" or "high frequency" 586 by pressing a button on a gamepad with their left or right index finger, respectively. The test tone 587 was embedded in a background of broadband noise (50-20000 Hz), and we titrated task difficulty by varying the sound level of the test tone (low frequency: 53, 57, 62, and $69 \mathrm{~dB}$ SPL; high frequency: $52,57,62$, and $69 \mathrm{~dB}$ SPL) relative to the noise ( $75 \mathrm{~dB}$ SPL). We mapped these signal-to-noise ratios (SNRs) onto a scale from $0.05-0.5$, based on the fractional voltage required

592 programming environment.

Most subjects completed 4-5 sessions of the task. Each session began with a set of 60-120 training trials. We then tested a different set of expectation-generating cues per session: "rulebased" cues, "stimulus-based" cues, or both (mixed condition; see Figure 1a). In a rule-based-cue

596 session (576 trials), we presented a visual cue at the beginning of each trial: a triangle pointing to 597 the left indicated 5:1 odds of a low-frequency test tone, a triangle pointing to the right indicated $598 \quad 5: 1$ odds of a high-frequency test tone, and a square indicated even (neutral) odds. The same cue was presented for a block of 48 trials and was varied randomly between blocks. 
600

601

602

603

604

605

606

607

608

609

610

611

612

613

614

615

616

617

618

619

620 $621=\$ 12)$.

In a stimulus-based-cue session (480 trials), we presented a tone-burst sequence (250 or 2000 Hz; 300-ms duration; 10-ms $\cos ^{2}$ ramp; 100-ms inter-burst interval) that preceded the test tone (i.e., the "pre-test" sequence). The pre-test sequence was always presented at the highest SNR. The length of the pre-test sequence varied between 2-14 tones, approximately exponentially distributed. These sequences were pseudorandomly generated, but we ensured that there were approximately the same number of high- and low-frequency tone bursts in each session.

There were also "mixed" sessions in which subjects had both rule-based and stimulus-based cues. In mixed-condition 1, which was divided into two sessions, we used the same rule-based cue (high or low prior) for the entire session (480/trials session) and varied the length of the pretest sequence. In mixed-condition 2 (576 trials), we randomly varied the rule-based cue and fixed the length of the pre-test sequence at five tone bursts.

Sessions were completed in the following order: rule-based, stimulus-based, mixed-condition 1 (using either a low- or high-prior cue, randomly selected), mixed-condition 1 (using the alternative prior cue), and mixed-condition 2. Because of attrition and other factors, not all subjects completed all conditions: rule-based, $N=49$ subjects completed the condition; stimulusbased, $N=45$; mixed-condition $1, N=41$, mixed-condition $2, N=21$. Further, for all subjects in the stimulus-based session and each session of mixed-condition 1, we discarded 38 trials that did not have a pre-test sequence (which were likely surprising and led to poor subject performance), leaving 442 trials for analysis. Also, one subject completed only 432 trials in the rule-based condition, and two subjects completed only 432 trials in mixed-condition 2. Subjects were paid $\$ 8$ plus a performance bonus per session. The bonus was $\$ 1$ for each $4 \%$ above $50 \%$ (max bonus 
623 Figure $1 \mathrm{~b}$ shows the timing of each trial. Subjects could make their response from the start of a

624 visual "go cue", which coincided with the initiation of the test tone, until the trial ended. Subjects

625 were given a 2-s response window, except for 25 subjects in the rule-based condition who were

626 given a 3-s response window. In mixed-condition 2, we did not provide a go cue because the

627 tone-burst sequence had a fixed, predictable duration and thus served as the go cue. Visual and

628 auditory feedback was provided after each trial indicating whether the subject's response was

629 correct or incorrect.

630 Data analysis

631 We conducted statistical analyses in R (R Core Team, 2020) and Matlab. Linear mixed-effects

632 models were fit using lme4 (Bates, Mächler, Bolker, \& Walker, 2015). When possible, we fit the

633 maximal model (i.e., random intercepts for subjects and random slopes for all within-subjects

634 variables). If the maximal model failed to converge or produced singular fits, we iteratively

635 reduced the random-effects structure until convergence (Bates, Kliegl, Vasishth, \& Baayen,

636 2015). Post-hoc comparisons were conducted using the emmeans package (Lenth, 2016). Post-

637 hoc multiple comparisons were corrected using the Bonferroni-Holm method (Holm, 1979).

638 We fit a logistic model to the rule-based and stimulus-based psychometric functions using a

639 maximum-likelihood approach. The logistic was of the form:

$$
P(H)=\lambda+\frac{1-2 \lambda}{1+e^{-f(x)}}
$$

640 where $P(H)$ is the probability that the subject chose high frequency, $f(X)$ is a linear function

641 predicting choice, and $\lambda$ is a lapse rate that sets the lower and upper asymptotes of the logistic

642 curve. 
644 For the rule-based condition, choice was predicted as:

649

645

646

647

648

650

660

651

652

653

654

655

656

657

658

659

661

662

663

664

$$
f(x)=\beta_{0}^{c}+\beta_{S N R} S N R,
$$

where $\beta_{S N R}$ determines the slope of the psychometric function, $S N R$ is the signed signal-to-noise ratio of the test tone (positive: high tones; negative: low tones), and $\beta_{0}^{c}$ determines the bias (offset). To capture the effects of the rule-based cues, we fit separate $\beta_{0}^{c}$ for each prior cue, $c$ (Low, Neutral, High). We also tested a model in which the slope was free to vary with prior cue.

For the stimulus-based condition, choice was predicted as:

$$
f(x)=\beta_{0}+\beta_{S N R} S N R+\sum_{i=1}^{6} \beta_{p t_{i}} p t_{i}+\sum_{i=1}^{6} \beta_{p t a_{i}} p t_{i}|S N R|
$$

where $p t_{i}$ is a pre-test tone burst at position $i$ numbered in reverse chronological order (i.e., $p t_{1}$ is the tone burst immediately prior to the test tone), $\beta_{p t_{i}}$ determines the bias (offset) attributable to each tone burst, and $\beta_{0}$ is a fixed offset capturing any idiosyncratic biases. The $p t_{i}$ were contrast coded such that $\beta_{p t_{i}}$ is the log-odds ratio of choosing high frequency over low frequency at a SNR of 0 (high-frequency regression contrast code: 0.5 ; low-frequency regression contrast code: -0.5$)$. We modeled a SNR-dependent adaptation-like effect as the interaction term $p t_{i}|S N R|:|S N R|$ is the unsigned signal-to-noise ratio of the test tone, and each $\beta_{p t a}$ determines how much each tone burst influences the slope of the psychometric function. We fit data only for the last six pre-test tone bursts because longer tone-burst sequences were infrequent.

\section{Model Comparison}

We assessed goodness-of-fit using Akaike information criteria (AIC). We also entered the AIC values into a Bayesian random-effects analysis, which attempts to identify the model among competing alternatives that is most prevalent in the population. This analysis yields a protected 
665

666

667

668

669

670

671

672

673

674

675

676

677

678

679

680

681

682

683

684

685

686

exceedance probability (PEP) for each model, which is the probability that the model is the most frequent in the population, above and beyond chance (Rigoux, Stephan, Friston, \& Daunizeau, 2014). PEPs were computed using the VBA toolbox (Daunizeau, Adam, \& Rigoux, 2014).

\section{Drift-diffusion modeling (DDM)}

We used PyDDM (Shinn et al., 2020) for all DDM analyses except for the simulations of the different types of biases, which were generated using an analytical solution to the DDM (Palmer et al., 2005). Unlike some of the fits described below, these simulations have a fixed bound and were not fit to the data but rather used ranges of parameter values that generated psychometric and chronometric functions that were qualitatively consistent with the data.

In the DDM framework, noisy evidence is accumulated until reaching one of the two bounds, triggering commitment to one of the two choices (in this case, low frequency or high frequency).

In our implementation, bound height was determined by a parameter $B$, which was equal to half the total distance between the bounds. All models, except as noted, included a linear collapsing bound, which accounts for the truncated RT distributions given that subjects were under time pressure. (RT was the time between onset of the go cue and gamepad button press, except for mixed-condition 2 in which a subject's RT was the time between test-tone offset and button press.) Thus, $B$ was the initial bound height, and an additional parameter $t_{B}$ determined the rate of linear collapse, such that total bound height at time $t$ was $2\left(B-t_{B} t\right)$.

The full slope or rate of evidence accumulation was defined as:

$$
\text { slope }=v_{S N R} S N R+\text { bias }_{\text {evidence }}+\text { adapt } *|S N R|,
$$

where $v_{S N R}$ is the drift rate, which influences the rate at which the sensory evidence provided by the test tone in the noisy background, $S N R$, contributes to evidence accumulation. 


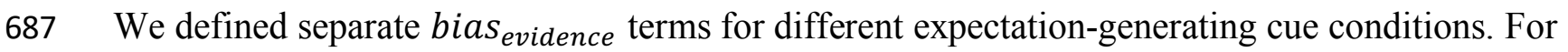
688 the rule-based condition, bias $_{\text {evidence }}$ was set to one of three parameters depending on the prior 689 cue (low: $v_{\text {Low }} ;$ neutral: $v_{0} ;$ high: $\left.v_{\text {High }}\right)$. For the stimulus-based condition, bias evidence $=$ $690 v_{0}+v_{\text {Bias }} p t_{\text {Bias }}$, where $v_{0}$ is a fixed offset capturing any idiosyncratic bias toward high or low 691 and $p t_{\text {Bias }}$ is an exponentially weighted sum of the pre-test tone bursts:

$$
p t_{\text {Bias }}=\sum_{i \in \text { high }} e^{-\frac{p t_{i}}{\tau_{\text {Bias }}}}-\sum_{j \in \text { low }} e^{-\frac{p t_{j}}{\tau_{\text {Bias }}}}
$$
where $p t_{i}$ and $p t_{j}$ are the sequence positions (numbered in reverse chronological order starting 694 at 0 ) of the high- and low-frequency tone bursts in the pre-test sequence, respectively; $\tau_{\text {Bias }}$ is a 695 time constant determining the decay in influence of the tone bursts with increasing temporal 696 distance from the test tone; and $v_{\text {Bias }}$ scales the total influence of the tone bursts on the rate of 697 evidence accumulation.

698 The full stimulus-based model also included an adaptation-like term in the slope, adapt * $699|S N R|$. The adapt quantity was defined as

$$
a d a p t=v a_{H i g h} \sum_{i \in h i g h} e^{-\frac{p t_{i}}{\tau_{v a}}}-v a_{\text {Low }} \sum_{j \in l o w} e^{-\frac{p t_{j}}{\tau_{v a}}}
$$
where $\tau_{v a}$ is a time constant and $v a_{H i g h}$ and $v a_{L o w}$ are separate weights for the high- and low-

702 frequency tone bursts.

703 Our model also controlled the location between the two bounds at which evidence starts 704 accumulating. If the starting point is not equidistant between the two bounds, less evidence is 705 required to make one choice versus the other; i.e., a starting-point bias. The starting point was 706 defined as 


$$
\text { starting point }=\text { bias }_{\text {start }} * 2 B \text {, }
$$

708

709

710

711

712

713

714

715

716

717

718

719

720

721

722

723

724

725

726

Finally, all models were fit with a lapse rate, $\lambda$, which mixed the RT distribution predicted by the

727

where $2 B$ is the total bound height, and bias start is the bias on the starting point, expressed as a fraction of total bound height $[0,1]$ (i.e., a value of 0.5 corresponds to an unbiased starting point, equidistant between the bounds). For the rule-based condition, bias start $_{\text {was }}$ set to one of three parameters depending on which prior cue was presented on that trial (low: $z_{\text {Low }} ;$ neutral: $z_{0} ;$ high: $\left.z_{\text {High }}\right)$. For the stimulus-based condition, bias $s_{\text {start }}=g\left(z_{0}+\right.$ $\left.z_{\text {Bias }} p t_{\text {Bias }}\right)$. The $p t_{\text {Bias }}$ was the same quantity as in the evidence-accumulation term, $z_{\text {Bias }}$ scaled the total influence of the tone bursts in the pre-test sequence on the starting point, and $z_{0}$ was a fixed offset accounting for idiosyncratic starting-point biases. The function $g(x)$ was the logistic function, $\frac{1}{1+e^{-x}}$, which constrained bias $s_{\text {start }}$ between 0 and 1 . We did not fit the rulebased condition using this logistic transformation; however, we transformed the resulting parameter estimates to the logit scale to be more comparable with the stimulus-based fits.

In addition to these parameters, all models were fit with a non-decision time, $n d t_{0}$, which accounted for the portion of RT not determined by decision formation (e.g., sensory or motor processing). For the stimulus-based condition, RTs were faster when the pre-test tone bursts were the same frequency. To account for this effect, total non-decision time was calculated as

$$
n d t=n d t_{0}+n d t_{\text {Bias }}\left|p t_{\text {Bias }}\right|
$$

where the extra additive term is the absolute value of the stimulus-based bias calculated above, weighted by $n d t_{\text {Bias }}$.
DDM with a uniform distribution in proportion to $\lambda$ (i.e., $\lambda=0.05$ implies that the final 
728 predicted distribution was a weighted average of 95\% the DDM distribution and 5\% a uniform

729

730

731

732

733

734

735

736

737

738

739

740

741

742

743

744

745

746

747

748

749 distribution).

We fit models separately to each task condition. See Shinn et al. (2020) for details of the optimization procedure. Models for the mixed conditions were identical to those described above but included both types of bias. For the rule- and stimulus-based conditions, we also fit reduced models to assess the necessity of different mechanisms for explaining subjects' behavior. The "base" model was a standard DDM augmented by idiosyncratic bias terms for evidence accumulation and starting point but without rule- or stimulus-based biases; it included six parameters, $v_{S N R}, B, v_{0}, z_{0}, n d t_{0}, \lambda$. The "collapsing bound" model included one additional parameter, $t_{B}$. The "full" model in the rule-based condition and the "stimulus-based bias" model in the stimulus-based condition included the bias evidence $_{\text {and }}$ bias $s_{\text {start }}$ (and $n d t_{\text {bias }}$ for the stimulus-based condition) terms, for a total of 11 parameters each. The "full" model in the stimulus-based condition further included the adapt term, for a total of 14 parameters.

We used the full-model fits to generate performance functions for each subject. The output of the performance function, $p c(z, v \mid c, \theta)$, was the predicted percentage of correct choices for a given combination of starting-point bias $(z)$ and evidence-accumulation bias $(v)$, given the expectationcue context ( $c$ ) and the subject's other best-fitting DDM parameters $(\theta)$. For the rule-based condition, the performance function was estimated for the context of the low prior and the high prior. For the stimulus-based condition, the context was set to either two low-frequency pre-test tone bursts (LL) or two high-frequency pre-test tone bursts $(\mathrm{HH})$. These tone bursts did not change the odds that the test tone would be low or high but nonetheless affected performance, as quantified via bias and adaptation-like effects in the DDM fits. 
750 We estimated the performance function across a grid of $z, v$ values $(z:(.1, .9) ; v:[-4,4])$, where

$751 z$ dictated the value of $z_{\text {Low }}$ or $z_{H i g h}$ in the rule-based condition and $z_{\text {Bias }}$ in the stimulus-based

752 condition, and $v$ dictated the value of $v_{\text {Low }}$ or $v_{\text {High }}$ in the rule-based condition and $v_{\text {Bias }}$ in the

753 stimulus-based condition. The predicted percent correct was generated from the DDM for each

754 point on the grid for all combinations of test-tone frequency value and SNR. Next, these values

755 were averaged according to the proportion of each trial type expected in that context. This

756 procedure was implemented separately for each subject, condition, and context. The resulting

757 performance functions were then normalized to a proportion of maximum possible performance

758 scale by dividing by the maximum accuracy obtained for each function. Performance functions

759 were then averaged across subjects for each context, and subject-averaged 97\% contours were

760 plotted using the Matlab contour function.

761 We fit a mixed-effects model to test whether the slope of the relationship between the starting-

762 point and evidence-accumulation biases differed between the rule- and stimulus-based

763 conditions. We entered the starting-point bias as the predictor and assessed the interaction

764 between starting-point bias and bias type $\left(z_{\text {High }}, z_{\text {Low }}\right.$, or $\left.z_{\text {Bias }}\right)$ in predicting the evidence-

765 accumulation bias. Specifically, we contrasted the slopes of the rule-based and stimulus-based

766 biases ([average of $z_{\text {High }}$ and $\left.\left.z_{\text {Low }}\right]-z_{\text {Bias }}\right)$ and the contrast of the rule-based slopes $\left(z_{\text {High }}-z_{\text {Low }}\right)$.

767 To confirm that the results were not dependent on which variable was entered as the outcome

768 and which as the predictor, we re-estimated the model with the evidence-accumulation bias as

769 predictor and starting point bias as outcome, which yielded similar results (rule-based vs.

770 stimulus-based: $B=0.47, t(53.65)=2.93, p=0.005$; rule-based high vs. low $(B=-0.21$,

$771 t(118.93)=-0.93, p>0.05)$. Because the rule- and stimulus-based biases are on different scales,

772 for both models, the bias variables were $z$-scored within condition. 
773 To test the association between rule-based and stimulus-based bias parameters, we calculated the

774 rule-based biases for a given condition as the difference between the high and low bias for both

775 evidence-accumulation (i.e., $v_{\text {High }}-v_{\text {Low }}$ ) and starting-point (i.e., $z_{\text {High }}-z_{\text {Low }}$ ) biases. We

776 then calculated the Spearman's correlation between these quantities and $v_{\text {Bias }}$ and $z_{\text {Bias }}$,

777 respectively.

\section{$778 \quad$ Pupillometry}

779 We recorded (EyeLink 1000 Plus; SR Research) each subject's right-eye position and pupil

780 diameter at a sampling rate of $1000 \mathrm{~Hz}$. Each subject maintained their gaze within a $14.66 \mathrm{x}$

$7818.11^{\circ}$ window throughout the trial.

782 If data were missing, either due to blinks or other artifacts, we linearly interpolated the pupil data 783 after removing $\pm 100 \mathrm{~ms}$ surrounding the blink (or artifact). We identified additional artifacts by

784 computing the difference between consecutive samples of the pupil time course and removed all 785 high-velocity periods (i.e., $>24$ a.u./ms). If the duration of any of these periods exceeded $16 \mathrm{ms,}$ 786 we also removed the $\pm 100 \mathrm{~ms}$ of surrounding data. These artifactual periods were then filled via

787 linear interpolation. We also interpolated gaze-position data for time points missing or removed 788 from the pupil time course. We excluded trials in which $>50 \%$ of the pupil data and sessions in 789 which $>60 \%$ of the pupil data were missing or were artifactual from further pupil analysis.

790 Additionally, in mixed-condition 1, both sessions had to pass these criteria for the subject to be 791 included in the pupil analysis.

792 The pupil time course was then low-pass filtered with an 8-Hz cutoff. We z-scored each subject's 793 time course within each block. For every trial, we calculated the baseline pupil diameter as the 
794 average diameter from $0-40 \mathrm{~ms}$ relative to test-tone onset, which was then subtracted from the 795 trial time course.

796 Gaze position for each trial was centered on the average gaze position during the baseline period.

797 For each session, we defined the fixation window as a circle containing $95 \%$ of gaze samples

798 across all data (1.36-3.29 visual angle across sessions). To minimize the impact of eye

799 movements on pupil diameter, we excluded trials in which gaze deviated from this window for

$800>15 \mathrm{~ms}$.

801 Finally, we excluded subjects with fewer than 75 trials remaining in a condition after all cleaning

802 procedures. Overall, we analyzed 44 subjects in the rule-based condition, 42 in the stimulus-

803 based condition, 33 in mixed-condition 1, and 17 in mixed-condition 2.

804 For statistical analyses, we downsampled the eye data to $50 \mathrm{~Hz}$. Like for the RT analyses, we 805 analyzed pupil diameter with respect to the congruence between the cue and choice and restricted

806 our main analyses to correct trials to avoid confounding changes in pupil size with

807 feedback/error related signals or off-task responses. We analyzed the rule- and stimulus-based

808 conditions by fitting a linear mixed-effects model to the data at every time point. These analyses

809 focused on contrasts of the pupil time course as a function of the congruency of choice with the

810 rule- and stimulus-based cues (e.g., incongruent - congruent), and the interaction of congruency

811 with $|\mathrm{SNR}|$. All models controlled for test-tone frequency, $|\mathrm{SNR}|$, baseline pupil diameter, and

812 gaze position. Data were aligned to choice (i.e., the time of the button press).

813 To test the association between behavioral bias and the congruency effects in the rule-based

814 condition, separate linear models were fit to the pupil data per subject to obtain estimates of

815 individual-subject congruency contrasts. These contrasts were then correlated across time with 
816 the estimate of behavioral bias obtained from the logistic fits to the psychometric function

817 described above, where bias was defined as $\beta_{0}^{\text {High }}-\beta_{0}^{\text {Low }}$. We used FDR correction to adjust $p$ -

818 values for multiple comparison across time (Benjamini \& Hochberg, 1995; Yekutieli \&

819 Benjamini, 1999). Congruency contrasts were corrected together across time and contrast. 


\section{Acknowledgements}

822 YEC, JIG, and LSA received support for this work from an Office of Naval Research grant

823 [N000141612539]. NT was supported by a T32 training grant from the National Institutes of

824 Health [MH014654].

\section{Competing interests}

826 JIG: Senior editor, eLife. The authors declare that they have no other competing interests.

\section{Data availability}

828 The datasets generated and analyzed for this article will be made available prior to publication at:

829 https://osf.io/f9nyr/. The analysis code for this article will be made available prior to publication

830 at: https://github.com/TheGoldLab/Analysis_Tardiff_etal_AuditoryPriors. 
Alais, D., Orchard-Mills, E., \& Van der Burg, E. (2015). Auditory frequency perception adapts rapidly to the immediate past. Attention, Perception, and Psychophysics, 77(3), 896-906. https://doi.org/10.3758/s13414-014-0812-2

Aston-Jones, G., \& Cohen, J. D. (2005). An integrative theory of locus coeruleus-norepinephrine function: adaptive gain and optimal performance. Annual Review of Neuroscience, 28(1), 403-450. https://doi.org/10.1146/annurev.neuro.28.061604.135709

Bastos, A. M., Lundqvist, M., Waite, A. S., Kopell, N., \& Miller, E. K. (2020). Layer and rhythm specificity for predictive routing. Proceedings of the National Academy of Sciences of the United States of America, 117(49), 31459-31469. https://doi.org/10.1073/pnas.2014868117

Bastos, A. M., Usrey, W. M., Adams, R. A., Mangun, G. R., Fries, P., \& Friston, K. J. (2012). Canonical Microcircuits for Predictive Coding. Neuron, 76(4), 695-711. https://doi.org/10.1016/j.neuron.2012.10.038

Bates, D., Kliegl, R., Vasishth, S., \& Baayen, H. (2015). Parsimonious Mixed Models. https://doi.org/arXiv:1506.04967

Bates, D., Mächler, M., Bolker, B., \& Walker, S. (2015). Fitting linear mixed-effects models using lme4. Journal of Statistical Software, 67(1), 1-48. https://doi.org/10.18637/jss.v067.i01

Benjamini, Y., \& Hochberg, Y. (1995). Controlling the False Discovery Rate: A Practical and Powerful Approach to Multiple Testing. Journal of the Royal Statistical Society: Series B (Methodological), 57(1), 289-300. https://doi.org/10.1111/j.2517-6161.1995.tb02031.x

Bouret, S., \& Sara, S. J. (2005). Network reset: A simplified overarching theory of locus coeruleus noradrenaline function. Trends in Neurosciences, 28(11), 574-582. https://doi.org/10.1016/j.tins.2005.09.002

Carbajal, G. V., \& Malmierca, M. S. (2018). The Neuronal Basis of Predictive Coding Along the Auditory Pathway: From the Subcortical Roots to Cortical Deviance Detection. Trends in Hearing, 22, 1-33. https://doi.org/10.1177/2331216518784822

Chambers, C., Akram, S., Adam, V., Pelofi, C., Sahani, M., Shamma, S., \& Pressnitzer, D. (2017). Prior context in audition informs binding and shapes simple features. Nature Communications, 8. https://doi.org/10.1038/ncomms 15027

Chambers, C., \& Pressnitzer, D. (2014). Perceptual hysteresis in the judgment of auditory pitch shift. Attention, Perception, and Psychophysics, 76(5), 1271-1279. https://doi.org/10.3758/s13414-014-0676-5

Dahmen, J. C., Keating, P., Nodal, F. R., Schulz, A. L., \& King, A. J. (2010). Adaptation to Stimulus Statistics in the Perception and Neural Representation of Auditory Space. Neuron, 66(6), 937-948. https://doi.org/10.1016/j.neuron.2010.05.018

Daunizeau, J., Adam, V., \& Rigoux, L. (2014). VBA: A probabilistic treatment of nonlinear models for neurobiological and behavioural data. PLoS Computational Biology, 10(1), 
e1003441. https://doi.org/10.1371/journal.pcbi.1003441

de Gee, J. W., Colizoli, O., Kloosterman, N. A., Knapen, T., Nieuwenhuis, S., Donner, T. H., ... Matthews, P. (2017). Dynamic modulation of decision biases by brainstem arousal systems. ELife, 6, 1127-1141. https://doi.org/10.7554/eLife.23232

de Gee, J. W., Knapen, T., \& Donner, T. H. (2014). Decision-related pupil dilation reflects upcoming choice and individual bias. Proceedings of the National Academy of Sciences of the United States of America, 111(5), E618-25. https://doi.org/10.1073/pnas.1317557111

de Gee, J. W., Tsetsos, K., Schwabe, L., Urai, A. E., McCormick, D., McGinley, M. J., \& Donner, T. H. (2020). Pupil-linked phasic arousal predicts a reduction of choice bias across species and decision domains. ELife, 9. https://doi.org/10.7554/eLife.54014

de Lange, F. P., Heilbron, M., \& Kok, P. (2018). How Do Expectations Shape Perception? Trends in Cognitive Sciences, 22(9), 764-779. https://doi.org/10.1016/J.TICS.2018.06.002

Doi, T., Fan, Y., Gold, J. I., \& Ding, L. (2020). The caudate nucleus contributes causally to decisions that balance reward and uncertain visual information. ELife, 9, 568733. https://doi.org/10.7554/eLife.56694

Dunovan, K. E., Tremel, J. J., \& Wheeler, M. E. (2014). Prior probability and feature predictability interactively bias perceptual decisions. Neuropsychologia, 61(1), 210-221. https://doi.org/10.1016/J.NEUROPSYCHOLOGIA.2014.06.024

Einhäuser, W., Koch, C., \& Carter, O. L. (2010). Pupil dilation betrays the timing of decisions. Frontiers in Human Neuroscience, 4, 18. https://doi.org/10.3389/fnhum.2010.00018

Fan, Y., Gold, J. I., \& Ding, L. (2018). Ongoing, rational calibration of reward-driven perceptual biases. ELife, 7. https://doi.org/10.7554/eLife.36018

Filipowicz, A. L. S., Glaze, C. M., Kable, J. W., \& Gold, J. I. (2020). Pupil diameter encodes the idiosyncratic, cognitive complexity of belief updating. ELife, 9. https://doi.org/10.7554/eLife.57872

Fischer, J., \& Whitney, D. (2014). Serial dependence in visual perception. Nature Neuroscience 2014 17:5, 17(5), 738-743. https://doi.org/10.1038/nn.3689

Fritsche, M., Spaak, E., \& de Lange, F. P. (2020). A Bayesian and efficient observer model explains concurrent attractive and repulsive history biases in visual perception. ELife, 9 . https://doi.org/10.7554/eLife.55389

Gelbard-Sagiv, H., Magidov, E., Sharon, H., Hendler, T., \& Nir, Y. (2018). Noradrenaline Modulates Visual Perception and Late Visually Evoked Activity. Current Biology, 28(14), 2239-2249.e6. https://doi.org/10.1016/j.cub.2018.05.051

Giangrand, J., Tuller, B., \& Kelso, J. A. S. (2003). Perceptual Dynamics of Circular Pitch. Music Perception, 20(3), 241-262. https://doi.org/10.1525/mp.2003.20.3.241

Gifford, A. M., Cohen, Y. E., \& Stocker, A. A. (2014). Characterizing the Impact of Category Uncertainty on Human Auditory Categorization Behavior. PLoS Computational Biology, 10(7). https://doi.org/10.1371/journal.pcbi.1003715 
Glaze, C. M., Kable, J. W., \& Gold, J. I. (2015). Normative evidence accumulation in unpredictable environments. ELife, 4(AUGUST2015). https://doi.org/10.7554/eLife.08825

Hanks, T. D., Mazurek, M. E., Kiani, R., Hopp, E., \& Shadlen, M. N. (2011). Elapsed decision time affects the weighting of prior probability in a perceptual decision task. Journal of Neuroscience, 31(17), 6339-6352. https://doi.org/10.1523/JNEUROSCI.5613-10.2011

Heilbron, M., \& Chait, M. (2018). Great Expectations: Is there Evidence for Predictive Coding in Auditory Cortex? Neuroscience, 389, 54-73. https://doi.org/10.1016/J.NEUROSCIENCE.2017.07.061

Heller, C. R., Schwartz, Z. P., Saderi, D., \& David, S. V. (2020, September 1). Selective effects of arousal on population coding of natural sounds in primary auditory cortex. BioRxiv. bioRxiv. https://doi.org/10.1101/2020.08.31.276584

Holm, S. (1979). A simple sequentially rejective multiple test procedure. Scandinavian Journal of Statistics, 6, 65-70. https://doi.org/10.2307/4615733

Holt, L. L., \& Lotto, A. J. (2008). Speech perception within an auditory cognitive science framework. Current Directions in Psychological Science, 17(1), 42-46. https://doi.org/10.1111/j.1467-8721.2008.00545.x

Joshi, S., \& Gold, J. I. (2020). Pupil size as a window on neural substrates of cognition. Trends in Cognitive Sciences, 24(6), 466-480. https://doi.org/10.1016/j.tics.2020.03.005

Joshi, S., Li, Y., Kalwani, R. M., \& Gold, J. I. (2016). Relationships between pupil diameter and neuronal activity in the locus coeruleus, colliculi, and cingulate cortex. Neuron, 89(1), 221234. https://doi.org/10.1016/j.neuron.2015.11.028

Kalwani, R. M., Joshi, S., \& Gold, J. I. (2014). Phasic Activation of Individual Neurons in the Locus Ceruleus/Subceruleus Complex of Monkeys Reflects Rewarded Decisions to Go But Not Stop. The Journal of Neuroscience, 34(41), 13656-13669. https://doi.org/10.1523/JNEUROSCI.2566-14.2014

Kelly, S. P., Corbett, E. A., \& O’Connell, R. G. (2020). Neurocomputational mechanisms of prior-informed perceptual decision-making in humans. Nature Human Behaviour, 5(4), 467-481. https://doi.org/10.1038/s41562-020-00967-9

Krishnamurthy, K., Nassar, M. R., Sarode, S., \& Gold, J. I. (2017). Arousal-related adjustments of perceptual biases optimize perception in dynamic environments. Nature Human Behaviour, 1(6), 0107. https://doi.org/10.1038/s41562-017-0107

Lenth, R. V. (2016). Least-squares means: The R package lsmeans. Journal of Statistical Software, 69(1), 1-33. https://doi.org/10.18637/jss.v069.i01

Lesicko, A. M. H., Angeloni, C. F., Blackwell, J. M., De Biasi, M., \& Geffen, M. N. (2021). Cortico-Fugal Regulation of Predictive Coding. BioRxiv, 2021.04.12.439188. https://doi.org/10.1101/2021.04.12.439188

Lieder, I., Adam, V., Frenkel, O., Jaffe-Dax, S., Sahani, M., \& Ahissar, M. (2019). Perceptual bias reveals slow-updating in autism and fast-forgetting in dyslexia. Nature Neuroscience, 22(2), 256-264. https://doi.org/10.1038/s41593-018-0308-9 
Lin, P.-A., Asinof, S. K., Edwards, N. J., \& Isaacson, J. S. (2019). Arousal regulates frequency tuning in primary auditory cortex. Proceedings of the National Academy of Sciences, 201911383. https://doi.org/10.1073/pnas.1911383116

McGinley, M. J., David, S. V., \& McCormick, D. A. (2015). Cortical Membrane Potential Signature of Optimal States for Sensory Signal Detection. Neuron, 87(1), 179-192. https://doi.org/10.1016/j.neuron.2015.05.038

McGinley, M. J., Vinck, M., Reimer, J., Batista-Brito, R., Zagha, E., Cadwell, C. R., ... McCormick, D. A. (2015). Waking state: Rapid variations modulate neural and behavioral responses. Neuron, 87(6), 1143-1161. https://doi.org/10.1016/j.neuron.2015.09.012

Mulder, M. J., Wagenmakers, E. J., Ratcliff, R., Boekel, W., \& Forstmann, B. U. (2012). Bias in the brain: A diffusion model analysis of prior probability and potential payoff. Journal of Neuroscience, 32(7), 2335-2343. https://doi.org/10.1523/JNEUROSCI.4156-11.2012

Näätänen, R., Paavilainen, P., Rinne, T., \& Alho, K. (2007). The mismatch negativity (MMN) in basic research of central auditory processing: A review. Clinical Neurophysiology, 118(12), 2544-2590. https://doi.org/10.1016/j.clinph.2007.04.026

Nelken, I. (2014). Stimulus-specific adaptation and deviance detection in the auditory system: experiments and models. Biological Cybernetics, 108(5), 655-663. https://doi.org/10.1007/s00422-014-0585-7

Palmer, J., Huk, A. C., \& Shadlen, M. N. (2005). The effect of stimulus strength on the speed and accuracy of a perceptual decision. Journal of Vision, 5(5), 1-1. https://doi.org/10.1167/5.5.1

Parras, G. G., Nieto-Diego, J., Carbajal, G. V., Valdés-Baizabal, C., Escera, C., \& Malmierca, M. S. (2017). Neurons along the auditory pathway exhibit a hierarchical organization of prediction error. Nature Communications, 8(1), 1-17. https://doi.org/10.1038/s41467-01702038-6

Pérez-González, D., Hernández, O., Covey, E., \& Malmierca, M. S. (2012). GABA A-mediated inhibition modulates stimulus-specific adaptation in the inferior colliculus. PLoS ONE, 7(3), e34297. https://doi.org/10.1371/journal.pone.0034297

R Core Team. (2020). R: A language and environment for statistical computing. Vienna, Austria: R Foundation for Statistical Computing. Retrieved from https://www.r-project.org

Ratcliff, R., \& McKoon, G. (2008). The diffusion decision model: Theory and data for twochoice decision tasks. Neural Computation, 20(4), 873-922. https://doi.org/10.1162/neco.2008.12-06-420

Reimer, J., Froudarakis, E., Cadwell, C. R., Yatsenko, D., Denfield, G. H., \& Tolias, A. S. (2014). Pupil fluctuations track fast switching of cortical states during quiet wakefulness. Neuron, 84(2), 355-362. https://doi.org/10.1016/j.neuron.2014.09.033

Reimer, J., McGinley, M. J., Liu, Y., Rodenkirch, C., Wang, Q., McCormick, D. A., \& Tolias, A. S. (2016). Pupil fluctuations track rapid changes in adrenergic and cholinergic activity in cortex. Nature Communications, 7(1), 13289. https://doi.org/10.1038/ncomms13289 
Rigoux, L., Stephan, K. E., Friston, K. J., \& Daunizeau, J. (2014). Bayesian model selection for group studies - Revisited. NeuroImage, 84, 971-985. https://doi.org/10.1016/j.neuroimage.2013.08.065

Schwartz, Z. P., Buran, B. N., \& David, S. V. (2020). Pupil-associated states modulate excitability but not stimulus selectivity in primary auditory cortex. Journal of Neurophysiology, 123(1), 191-208. https://doi.org/10.1152/jn.00595.2019

Schwiedrzik, C. M., Ruff, C. C., Lazar, A., Leitner, F. C., Singer, W., \& Melloni, L. (2014). Untangling perceptual memory: Hysteresis and adaptation map into separate cortical networks. Cerebral Cortex, 24(5), 1152-1164. https://doi.org/10.1093/cercor/bhs396

Seriès, P., \& Seitz, A. R. (2013). Learning what to expect (in visual perception). Frontiers in Human Neuroscience, 7(OCT), 1-14. https://doi.org/10.3389/fnhum.2013.00668

Shinn, M., Lam, N. H., \& Murray, J. D. (2020). A flexible framework for simulating and fitting generalized drift-diffusion models. ELife, 9, 1-27. https://doi.org/10.7554/ELIFE.56938

Shu, Z. J., Swindale, N. V., \& Cynader, M. S. (1993). Spectral motion produces an auditory after-effect. Nature, 364(6439), 721-723. https://doi.org/10.1038/364721a0

Snyder, J. S., Carter, O. L., Hannon, E. E., \& Alain, C. (2009). Adaptation Reveals Multiple Levels of Representation in Auditory Stream Segregation. Journal of Experimental Psychology: Human Perception and Performance, 35(4), 1232-1244. https://doi.org/10.1037/a0012741

Sotiropoulos, G., Seitz, A. R., \& Seris, P. (2011). Changing expectations about speed alters perceived motion direction. Current Biology, 21(21), R883-R884. https://doi.org/10.1016/J.CUB.2011.09.013

Stocker, A. A., \& Simoncelli, E. P. (2005). Sensory adaptation within a Bayesian framework for perception. In Advances in Neural Information Processing Systems (Vol. 18, pp. 12891296). MIT Press.

Summerfield, C., \& de Lange, F. P. (2014). Expectation in perceptual decision making: neural and computational mechanisms. Nature Reviews Neuroscience, 15(11), 745-756. https://doi.org/10.1038/nrn3838

Sussman, E. S. (2007). A new view on the MMN and attention debate: The role of context in processing auditory events. Journal of Psychophysiology, 21(3-4), 164-175. https://doi.org/10.1027/0269-8803.21.34.164

Symonds, R. M., Lee, W. W., Kohn, A., Schwartz, O., Witkowski, S., \& Sussman, E. S. (2017). Distinguishing Neural Adaptation and Predictive Coding Hypotheses in Auditory Change Detection. Brain Topography, 30(1), 136-148. https://doi.org/10.1007/s10548-016-0529-8

Todorovic, A., \& de Lange, F. P. (2012). Repetition suppression and expectation suppression are dissociable in time in early auditory evoked fields. Journal of Neuroscience, 32(39), 1338913395. https://doi.org/10.1523/JNEUROSCI.2227-12.2012

Todorovic, A., van Ede, F., Maris, E., \& de Lange, F. P. (2011). Prior expectation mediates neural adaptation to repeated sounds in the auditory cortex: An MEG study. Journal of 
Neuroscience, 31(25), 9118-9123. https://doi.org/10.1523/JNEUROSCI.1425-11.2011

1027

1028

1029

1030

1031

1032

1033

1034

1035

1036

1037

1038

1039

1040

1041

1042

1043

1044

1045

1046

1047

1048

1049

1050
Tsunada, J., Cohen, Y., \& Gold, J. I. (2019). Post-decision processing in primate prefrontal cortex influences subsequent choices on an auditory decision-making task. ELife, 8. https://doi.org/10.7554/eLife.46770.001

Urai, A. E., Braun, A., \& Donner, T. H. (2017). Pupil-linked arousal is driven by decision uncertainty and alters serial choice bias. Nature Communications, 8(1), 14637. https://doi.org/10.1038/ncomms 14637

Vinck, M., Batista-Brito, R., Knoblich, U., \& Cardin, J. A. (2015). Arousal and Locomotion Make Distinct Contributions to Cortical Activity Patterns and Visual Encoding. Neuron, 86(3), 740-754. https://doi.org/10.1016/j.neuron.2015.03.028

Waschke, L., Tune, S., \& Obleser, J. (2019). Local cortical desynchronization and pupil-linked arousal differentially shape brain states for optimal sensory performance. ELife, 8. https://doi.org/10.7554/eLife.51501

Wei, X. X., \& Stocker, A. A. (2015). A Bayesian observer model constrained by efficient coding can explain "anti-Bayesian" percepts. Nature Neuroscience, 18(10), 1509-1517. https://doi.org/10.1038/nn.4105

Yekutieli, D., \& Benjamini, Y. (1999). Resampling-based false discovery rate controlling multiple test procedures for correlated test statistics. Journal of Statistical Planning and Inference, 82(1-2), 171-196. https://doi.org/10.1016/s0378-3758(99)00041-5

Zhao, S., Chait, M., Dick, F., Dayan, P., Furukawa, S., \& Liao, H.-I. I. (2019). Pupil-linked phasic arousal evoked by violation but not emergence of regularity within rapid sound sequences. Nature Communications, 10(1), 4030. https://doi.org/10.1038/s41467-01912048-1 


\section{Supplementary Figures}

a

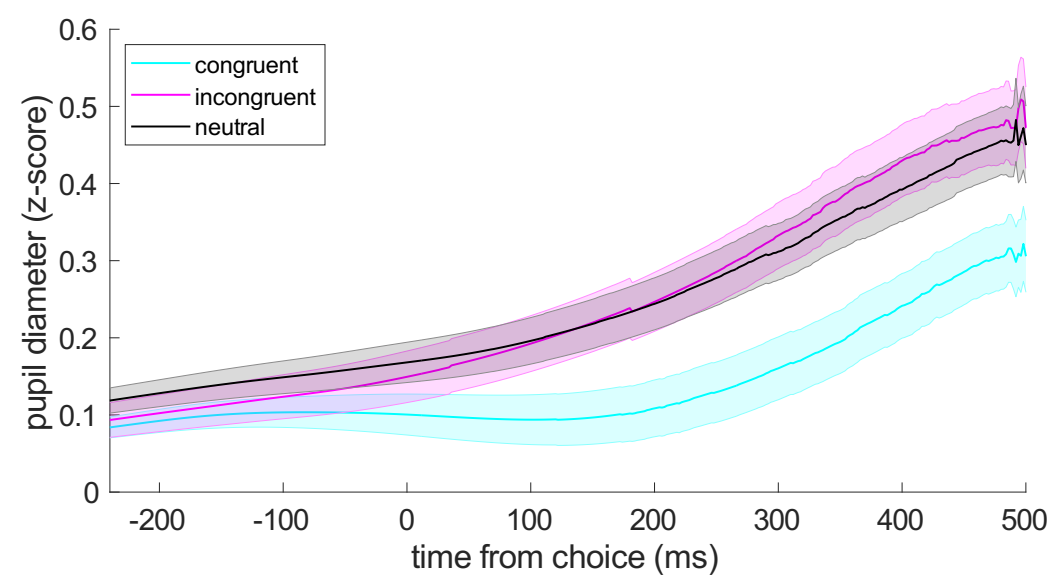

b

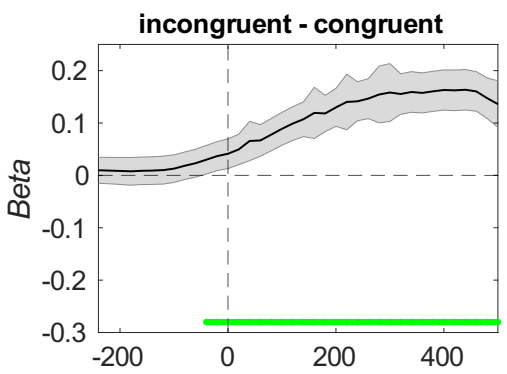

1052

1053

Figure 5-Figure supplement 1. Rule-based cues modulated pupil-linked arousal on

1054 incorrect trials. a Pupil diameter on incorrect trials, aligned to choice and plotted as a function

1055 of the congruence between the choice and the prior. Lines and error bars are means and SEM across all trials and subjects. b Regression coefficients (beta values) from a linear mixed-effects

1057 model as a function of different pupil contrasts (see labels above each panel). Error bars are 95\%

1058 confidence intervals of the parameter estimates. The time window in $\mathbf{a}$ and $\mathbf{b}$ begins earlier than the corresponding window from Figure 5 to focus on the time period in which the incongruent congruent effect was most prominent in the correct trials. The window ends earlier because incorrect trials were programmed to end faster than correct trials. Values in $\mathbf{b}$ were computed in 20 ms-wide time bins. Green lines indicate time bins for which $p_{\text {corrected }}<0.05$, FDR-corrected across time and contrast. 